\title{
Benchmarking of Monte Carlo flux simulations of electrons in $\mathrm{CO} 2$
}

Citation for published version (APA):

Vialetto, L., Viegas, P., Longo, S., \& Diomede, P. (2020). Benchmarking of Monte Carlo flux simulations of electrons in CO2. Plasma Sources Science \& Technology, 29(11), [115006]. https://doi.org/10.1088/13616595/abbac3

Document status and date:

Published: 01/11/2020

DOI:

10.1088/1361-6595/abbac3

Document Version:

Publisher's PDF, also known as Version of record

Document license:

Taverne

Please check the document version of this publication:

- A submitted manuscript is the version of the article upon submission and before peer-review. There can be important differences between the submitted version and the official published version of record.

People interested in the research are advised to contact the author for the final version of the publication, or visit the DOI to the publisher's website.

- The final author version and the galley proof are versions of the publication after peer review.

- The final published version features the final layout of the paper including the volume, issue and page numbers.

Link to publication

\footnotetext{
General rights rights.

- You may freely distribute the URL identifying the publication in the public portal. please follow below link for the End User Agreement:

www.umlib.nl/taverne-license

Take down policy

If you believe that this document breaches copyright please contact us at:

repository@maastrichtuniversity.nl

providing details and we will investigate your claim.
}

Copyright and moral rights for the publications made accessible in the public portal are retained by the authors and/or other copyright owners and it is a condition of accessing publications that users recognise and abide by the legal requirements associated with these

- Users may download and print one copy of any publication from the public portal for the purpose of private study or research.

- You may not further distribute the material or use it for any profit-making activity or commercial gain

If the publication is distributed under the terms of Article $25 \mathrm{fa}$ of the Dutch Copyright Act, indicated by the "Taverne" license above, 


\title{
Benchmarking of Monte Carlo flux simulations of electrons in $\mathrm{CO}_{2}$
}

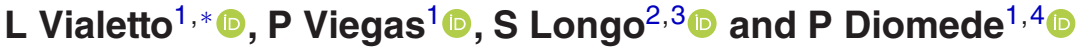 \\ ${ }^{1}$ Center for Computational Energy Research, DIFFER-Dutch Institute for Fundamental Energy Research, \\ De Zaale 20, 5612 AJ Eindhoven, The Netherlands \\ ${ }^{2}$ Dipartimento di Chimica, Università degli Studi di Bari, via Orabona 4, 70126 Bari, Italy \\ 3 Istituto per la Scienza e Tecnologia dei Plasmi, CNR, 70126 Bari, Italy \\ ${ }^{4}$ Faculty of Science and Engineering, Maastricht University, Paul Henri Spaaklaan 1, 6229 GS, \\ Maastricht, The Netherlands
}

E-mail: L.Vialetto@differ.nl

Received 11 June 2020, revised 28 August 2020

Accepted for publication 22 September 2020

Published 10 November 2020

\begin{abstract}
Electron velocity distribution functions (EVDFs) in $\mathrm{CO}_{2}$ obtained by means of the Monte Carlo flux (MCF) method are compared with results from two-term and multi-term Boltzmann solvers. The MCF method provides detailed calculations of the EVDF through a highly efficient variance reduction technique. Benchmark calculations of Legendre polynomial coefficients of the EVDF expansion are reported for a wide range of reduced electric fields $(E / N)$, showing excellent agreement with multi-term solutions. Rate coefficients of inelastic processes calculated from two-term Boltzmann solvers differ significantly, up to $70 \%$, from MCF and multi-term solutions, due to the anisotropy of the EVDF. An extension of the method to consider the thermal distribution of the background gas is also presented. This extension, together with an accurate description of the population of rotationally and vibrationally excited states, provides excellent agreement with measured transport coefficients at low $E / N$. A good agreement is obtained at moderate $E / N$ between experimental values of dissociation rate coefficients and MCF calculations after careful consideration and analysis of several cross sections data sets.
\end{abstract}

Keywords: Monte Carlo flux, $\mathrm{CO}_{2}$, electron velocity distribution function, benchmarking

(Some figures may appear in colour only in the online journal)

\section{Introduction}

The investigation of electron kinetics and transport in $\mathrm{CO}_{2}$ is an important topic, since collisions of free electrons with $\mathrm{CO}_{2}$ molecules are fundamental for the excitation of internal degrees of freedom and electronic states, dissociation and ionization of the molecule [1]. Initial studies on this topic focussed on the description of laser kinetics [2-4] and plasma activation of small molecules, such as $\mathrm{CO}_{2}[5,6]$. More recently, renewed interest on the topic has been mainly motivated by a possible application to carbon capture and utilization [7-9]. In terms of numerical investigations of electron kinetics, $\mathrm{CO}_{2}$ is

* Author to whom any correspondence should be addressed. considered a complex gas due to the presence of a broad Ramsauer minimum in the elastic momentum transfer cross section, together with evenly distributed electron energy loss processes due to vibrational or electronic excitations. In the pioneering work of Nighan [10], emphasis has been given to the evaluation of the electron energy distribution function (EEDF) that exhibits deviations from the Boltzmann one, upon application of an external and homogeneous electric field.

Detailed information about past and present advances in the solution of the electron Boltzmann equation (EBE) can be found in [11-14]. A common approach for the solution of the EBE is the expansion of the electron velocity distribution function (EVDF) in spherical harmonics for the angular dependence of the velocity space. Instead of the full spherical harmonics representation, an expansion in Legendre 
polynomials can be applied in cases of rotational symmetry of the distribution function around the direction of the electric field. A considerable simplification is often obtained by assuming that the EVDF is well represented by the first two terms in the expansion. This is referred in literature as twoterm approximation [15]. Recently, progress in the modelling and understanding of $\mathrm{CO}_{2}$ plasmas has been made by several groups using the two-term approach. The kinetics of $\mathrm{CO}_{2}$ dissociation kinetics has been studied by Bogaerts and co-authors [16-18]. In those models, the effects of several chemical processes are taken into account in a OD approach, by means of the codes ZDPlasKin [19] or GLOBAL_KIN [20, 21]. In particular, in ZDPlasKin, rate coefficients of electron impact processes are computed with the two-term solver BOLSIG+ $[22,23]$. A detailed investigation of electron kinetics has been performed by the Bari group [24, 25] with the use of native codes that couple a time resolved solution of the two-term EBE with the description of vibrational and chemical kinetics. Efforts in coupling a solution of the two-term EBE with the rate equations for vibrational and chemical kinetics have been carried out also by the Lisbon group, with particular emphasis on the comparison with experimental analysis of glow discharges $[26,27]$. In addition, a complete set of cross sections has been proposed by the Lisbon group [28] and optimized for the calculation of flux transport coefficients in swarm analysis with the two-term solver LoKI-B, that has been recently released as an open source code $[29,30]$. However, the two-term approximation obtained by an expansion in Legendre polynomials is not applicable in absence of rotational symmetry in the velocity space [31]. In addition, the anisotropy of the distribution is enhanced if inelastic processes give a significant contribution to electron energy losses [32]. The two-term approach can be extended to higher orders in the expansion, giving rise to multi-term Boltzmann solvers, that are typically applied to accurate calculations of electron transport coefficients [13]. Another motivation for accurate solutions of the EBE is the calculation of transport coefficients (bulk and flux) that have been investigated in the framework of swarm analysis experiments $[33,34]$. Such multi-term solvers are known to overcome the small anisotropy assumption implicit in the two-term approach. An example of open source code that is based on a multi-term expansion in Legendre polynomials of the EVDF is MultiBolt [35, 36]. Multi-term solutions of the EBE to the $\mathrm{CO}_{2}$ case have been performed by Loffhagen [37] for the study of spatial and temporal relaxation of the EVDF. In addition, the group at James Cook University has considered the effect of magnetic fields on electron transport $[11,38]$, pointing out the necessity of a full spherical harmonics expansion in cases of absence of rotational symmetry in the velocity space. A comparison of different methods to calculate electron swarm coefficients has been presented in Segur et al [39] and, more recently, in Vass et al [40] for a wide range of reduced electric fields $(E / N)$, this last using the IST-Lisbon set of cross sections [28]. Monte Carlo (MC) methods can also be applied for solving the charged particles transport problem $[41,42]$, especially in conditions of strong anisotropy of the distribution function in the velocity space or when spatially dependent quantities are needed [15]. Since the MC method is mainly based on the description of electron history from collision to collision, it is of easier implementation than a multi-term solver. Moreover, the method is equivalent to an infinite expansion in spherical harmonics [43]. Recently, electron impact rate coefficients for vibrational excitation of $\mathrm{CO}_{2}$ gas molecules have been calculated using an MC method by Vojnović et al [44], in the presence of DC electric and magnetic fields. In general, application of particle based methods is favoured in plasmas due to the complexity of these last. As an example, a recent work has proposed to study the splitting of $\mathrm{CO}_{2}$ in a plasma-enhanced catalysis system by means of a 1D particle-in-cell with Monte Carlo collisions (PIC/MCC) code [45]. An example of code based on $\mathrm{MC}$ and used for calculation of electron transport properties is Magboltz, that was developed by Biagi [46, 47]. Considerable effort has been devoted to benchmarking Boltzmann solvers and MC codes for electrons. In particular, notable is the work of Braglia and Romanó [48] on the calculations of electron transport coefficients in $\mathrm{CO}_{2}$. An extended comparison, including also multi-term calculations, has been published subsequently by Braglia, Wilhelm and Winkler [49], highlighting the inadequacy of the two-term approach for the case of a spatially homogeneous system under the application of a constant electric field. In spite of the excellent agreement between MC and Boltzmann six-term calculations, in [49] the difficulties of obtaining accurate MC calculations of excitation rates, particularly when they are sensitive to the tail of the distribution, are also pointed out. This is mainly due to stochastic fluctuations in the computed EEDFs that affect the accuracy of MC results. Furthermore, MC calculations may require considerable computational time, due to the high number of electron collisions that have to be simulated before steady-state is attained. Moreover, being the method intrinsically timedependent, it can suffer from a problem of multiple time scales that range from the inverse of the momentum relaxation frequency to the inverse of the energy relaxation frequency. The ratio of the two time scales, for the case of electron transport, is of the order of the electron to heavy particle mass ratio $m / M[1]$.

Acceleration of the convergence and improvement of MC efficiency and accuracy can be obtained by means of variance reduction techniques (VRTs) [50, 51]. Those techniques are usually employed in MC to reduce the statistical error in the calculation of macroscopic quantities by using different statistical weights for the simulated particles. An example of VRT is the Monte Carlo flux (MCF). The MCF method has been originally proposed by Schaefer and Hui [52] for studying the electron transport problem and applied afterwards mainly by Longo and Capitelli $[15,53]$. In the MCF method, while collisional events are still based on an MC description, a reduction of the computational time is obtained by employing an efficient VRT. Moreover, the problem of strongly different timescales is solved by the use of a deterministic Markov chain for the time evolution. However, as the method has generally been overlooked, it was never benchmarked against solutions of the EBE under the simplest stationary and homogeneous conditions for molecular gases. The present work is an extension of previous studies, like [49], focussing here on a systematic comparison of different numerical methods to describe electrons 
in $\mathrm{CO}_{2}$. A Fortran version of MCF has been recently implemented and benchmarked for electrons in argon by the present authors [54]. This reconsideration is in the perspective of an integration of $\mathrm{MCF}$ in $0 \mathrm{D} / 1 \mathrm{D}$ codes describing the interplay between electrons and chemical kinetics in plasmas.

In particular, considering electron transport in gases, often the cold gas approximation is used, assuming a fixed background of target particles at rest. However, this approximation is not valid for low reduced electric fields $(E / N)$, when the mean electron energy is comparable with the thermal energy of the background gas. Extension of the Boltzmann transport equation to consider energy exchange in elastic collision due to the thermal motions of molecules in a DC electric field has been originally considered by Davydov. This correction term in the elastic operator, also called Davydov-Boltzmann term [13], can be derived by an analysis of the linear EBE under assumption of conservative collisions, expansion of the distribution function in the first order and thermal equilibrium of the background gas molecules [55] or by considering the analogy with a Fokker-Planck formulation [56]. In this work, the MCF method is extended to consider the contribution of finite gas temperature of the background gas by means of an exact test particle MC technique [57]. This extension allows one to study gas kinetics effects of electron transport at low $E / N$. Moreover, considering the specific case of $\mathrm{CO}_{2}$, inconsistencies arising in neglecting the population of internal degrees of freedom, while including effects of finite gas temperature, are highlighted. Beside the scope of this work, another interesting application where MCF can be advantageous compared with other EBE solvers, is in near-runaway conditions. In fact, MCF can provide fast and accurate calculations of EVDFs at high $E / N$.

The paper is organized as follows. In section 2, a description of the numerical method is introduced. Section 3.1 presents results of benchmarked calculations of EEDFs and anisotropic components of the EVDF, over a wide range of $E / N$. The Boltzmann solvers BOLSIG+ [23] and MultiBolt [35], using a two-term and ten-term expansion in Legendre polynomial, respectively, are used for benchmarking. Particular emphasis is given to the accuracy of calculation of rate coefficients of inelastic processes. In section 3.2, an upgrade of the MCF method, with the technique that takes into account the thermal velocity distribution of the background gas, is presented. In section 3.3, effects of gas kinetics and population of rotationally and vibrationally excited states are analysed for electrons in $\mathrm{CO}_{2}$. The importance of considering accurate descriptions of excited states population is also emphasized. In section 3.4, the use of Biagi cross sections is tested for calculation of transport coefficients and electron impact dissociation rate coefficients and compared with experimental measurements.

\section{Numerical method}

The numerical method has been described in detail in [54]. Here we report the main characteristics of the method and code implementation and the new features added in the present work. The case of a spatially homogeneous system, in the presence of an external DC electric field applied along the $\vec{z}$-direction is considered. The MCF method [52] is used for calculation of EVDFs in pure $\mathrm{CO}_{2}$. In MCF calculations, the velocity space is partitioned into a finite number of cells of size $\Delta \vec{v}$ in the $\vec{v}$-direction. Hence the electron velocity distribution is obtained as a distribution defined in a discrete space. Usually, under condition of spherical symmetry around the direction of the electric field, such as in the present work, a $2 \mathrm{D}$ discretization in energy and $\cos \theta$ is sufficient for a characterization of the velocity distribution function. Furthermore, this description allows one to rewrite the electron transport problem in the following form [15]:

$$
n_{i}(t+\Delta t)=\sum_{j} q_{j i}(\Delta t) n_{j}(t)-n_{i}(t) \sum_{j} q_{i j}(\Delta t),
$$

where $n_{i}(t)$ is the number of electrons in the $i$ th cell at time $t, q_{j i}(\Delta t)$ is the conditional transition probability of electrons moving from the $i$ th to the $j$ th cell in velocity space within the time interval $\Delta t$. In order to have an accurate description of the collisional transport in velocity space, $\Delta t$ is assumed to have values that ensure enough 'mixing' of the velocity components due to scattering events, such that:

$$
\tau_{\mathrm{m}}<\Delta t \ll \tau_{\epsilon},
$$

where $\tau_{\mathrm{m}}$ is the momentum relaxation time and $\tau_{\epsilon}$ is the energy relaxation time. In this way, the linear Boltzmann equation for electrons can be described as a discrete Markov process where the time evolution of the system depends only on the knowledge of accurate transition probabilities [58]. In $\mathrm{MCF}$, these last are calculated with short time MC simulations that track electron trajectories from the initial to the final cell that is reached after $\Delta t$. Moreover, the electron motion is followed for a time typically much shorter than the relaxation time of the distribution function (equation (2)). This makes MCF much more computationally efficient than a conventional MC method.

The numerical implementation of MCF has been performed in Fortran and it includes three main modules that are called in the following order:

- Discretization module: this module is responsible for reading and parsing the MCF input data. In fact, the code requires as input collisional cross sections in LXCat format [59]. In addition, numerical parameters such as energy bin size $(\Delta \epsilon)$, maximum energy $\left(\epsilon_{\max }\right)$, number of $\cos \theta$ bins $\left(n_{\cos \theta}\right)$ and number of electrons per cell $\left(n_{\mathrm{p}}\right)$ are required. Cross sections are interpolated in the energy range $\left[0, \epsilon_{\max }\right]$ using a piecewise linear interpolation. The EVDF and quantities averaged over it are calculated for different physical parameters, such as reduced electric field $(E / N)$, initial gas composition and gas temperature $\left(T_{\text {gas }}\right)$ that, in this work, are assumed to be constant.

- MC module: in this module, transition probabilities are calculated with MC simulations that take into account an exact description of collision statistics with the modified time step approach [42], where each particle contributes to the statistical sampling of transition probabilities [52]. The calculation is initiated by inserting in each cell a 
uniform distribution of $n_{\mathrm{p}}$ electrons, whose trajectory is traced for a time interval $\Delta t$. The time interval is estimated using the criterion in [54]. This heuristic procedure has been proven to give values of $\Delta t$ that satisfy condition (2). Pseudo-random numbers from a Fortran implementation of the Mersenne Twister algorithm [60, 61] are used to compute the time between subsequent collisions and the type of collision with the null-collision method [62]. Depending on the nature of the collision, random numbers are also used to compute the electron energy and direction after the scattering [42, 63]. Effects of non-conservative collisions are included using a dynamic list of particles, as described in [54].

As a difference with respect to [54], two different treatments for the electron velocity distribution of the background gas are considered. If the cold gas approximation is used, the background of $\mathrm{CO}_{2}$ molecules is considered at rest. In this case, the collision frequency for an electron of velocity $\vec{v}$ is calculated as

$$
\nu(v)=N \sigma(v) v,
$$

where $N$ is the gas number density, $\sigma(v)$ is the collisional cross section for electron-molecule scattering and $v$ is the electron speed. Equation (3) is usually a good approximation to describe electron transport in gases because of the large electron to heavy particles mass difference and the high electron to neutral temperature ratio, but it may become inadequate at low values of reduced electric field, such that the mean electron energy becomes comparable with the thermal energy of the gas. For this reason, the effect of a finite temperature of the background gas is taken into account following a procedure introduced in [57]. According to this treatment, for each electron collision, the velocity of the neutral background gas $\vec{u}$ is sampled from a Maxwell-Boltzmann distribution function at $T_{\text {gas. }}$. In this way, the electron collision frequency can be written in the exact form, depending on the electron-neutral relative velocity [64]:

$$
\nu(v)=N \int \sigma(|\overrightarrow{\mathrm{v}}-\vec{u}|)|\overrightarrow{\mathrm{v}}-\vec{u}| F(\vec{u}) \mathrm{d} \vec{u},
$$

where $F(\vec{u})$ is the distribution of the $\mathrm{CO}_{2}$ molecules at $T_{\text {gas }}$. The von Neumann rejection technique is used to sample the velocity components of the vector $\vec{u}$.

- Markov chain module: in this module, transition probabilities from MC simulations are stored in the form of a matrix. If time dependent quantities are sought, equation (1) is solved iteratively in the form of a matrix equation. As an alternative, the steady-state solution is retrieved by solving an eigenvalue problem using the DGEEV subroutine of the LAPACK 3.8.0 library [65].

As output, MCF provides time-dependent or steady-state EVDFs which are used for the computation of Legendre polynomial coefficients, rate coefficients and transport coefficients.

\section{Results}

Electron impact cross sections with $\mathrm{CO}_{2}$ molecules are taken from the Biagi database [66], that includes 92 different collision processes. This cross sections set has been recently transcribed from the code Magboltz v11.6 [47] and carefully checked by comparing Boltzmann and MC calculations [66]. In particular, the dataset provides an elastic momentum transfer cross section that takes into account the contribution from the ground vibrational state $\mathrm{CO}_{2}\left(\nu_{1} \nu_{2} \nu_{3}=000\right.$ ) (where $\nu_{1}$, $\nu_{2}$ and $\nu_{3}$ are the vibrational quantum number of the symmetric stretching, bending and asymmetric stretching mode, respectively), including rotational states and vibrational bending mode in thermal equilibrium at $293.15 \mathrm{~K}$. The elastic momentum transfer cross section for the vibrational bending mode is tabulated separately and available in the same database. Such a cross section takes into account the contribution of the first level of the bending mode $\left(\mathrm{CO}_{2}(010)\right)$, together with two low-lying vibrational levels $\left(\mathrm{CO}_{2}(020)\right.$ and $\left.\mathrm{CO}_{2}(030+110)\right)$. For electron energies below $5 \mathrm{eV}$, inelastic processes are dominated by electron impact vibrational excitation of $\mathrm{CO}_{2}(000)$ that is described by a set of 14 processes. In particular, the following processes are included:

- Vibrational excitation of $\mathrm{CO}_{2}(000)$ to bending mode levels with thresholds $0.083,0.159$ and $0.251 \mathrm{eV}$, corresponding to the states $\mathrm{CO}_{2}(010), \mathrm{CO}_{2}(020)$ and $\mathrm{CO}_{2}(030+110)$.

- Vibrational excitation of $\mathrm{CO}_{2}(000)$ to the first vibrational level of the symmetric stretching mode with threshold $0.172 \mathrm{eV}$ (i.e. $\mathrm{CO}_{2}(100)$ ).

- Vibrational excitation of $\mathrm{CO}_{2}(000)$ to the first vibrational level of the asymmetric stretching mode with threshold $0.291 \mathrm{eV}$ (i.e. $\left.\mathrm{CO}_{2}(001)\right)$.

In addition to the processes above, electron impact excitations to vibrational states with a threshold up to $2.5 \mathrm{eV}$ are taken into account. For higher electron energies, other inelastic conservative processes are described by 79 reactions that include electron impact excitation and dissociation. Cross sections for those processes are derived mainly from the analysis of photoabsoption in $\mathrm{CO}_{2}$ [67]. This technique gives cross sections for levels that are coupled with the ground electronic state through dipole excitations [68]. Non-dipole allowed transitions are called triplet excitations and related cross sections are optimized in Magboltz [47] to reproduce the measured Townsend ionization coefficient [68]. In particular, for electron energies between 6 and $12 \mathrm{eV}$, the following processes contribute to electron impact dissociation of $\mathrm{CO}_{2}$ :

- Dipole allowed transitions to singlet states leading to dissociation of $\mathrm{CO}_{2}$ into $\mathrm{CO}$ and $\mathrm{O}$, described by 10 different processes with thresholds ranging from 6.50 to $8.75 \mathrm{eV}$

- Dissociative excitation via $\mathrm{CO}_{2}$ triplet state with threshold $8.89 \mathrm{eV}$

- A second group of dipole transitions to singlet states leading to dissociation of $\mathrm{CO}_{2}$ into $\mathrm{CO}$ and $\mathrm{O}$, described by 6 
different processes with thresholds ranging from 8.90 to $10.15 \mathrm{eV}$.

- Dissociative excitation with threshold $11.05 \mathrm{eV}$.

- Dissociative excitation via sum of triplets with a threshold of $11.30 \mathrm{eV}$.

Electron impact dissociation of $\mathrm{CO}_{2}$ is also considered at energies above $13.7 \mathrm{eV}$, that is the ionization energy of $\mathrm{CO}_{2}$ into $\mathrm{CO}_{2}^{+}$. In particular, the following processes are included:

- Dipole allowed transitions leading to dissociation, that are separated from ionization processes based on the average of many measurements of the ionization efficiency $[67,68]$, described by 25 different processes with thresholds ranging from 13.78 to $19.75 \mathrm{eV}$.

- Dissociative excitation via sum of triplets with a threshold of $25 \mathrm{eV}$.

Ionization processes of $\mathrm{CO}_{2}$ to different singly-charged and multiply-charged ions $\left(\mathrm{CO}_{2}^{+}\left(\tilde{A}^{2} \Pi_{u}\right), \mathrm{CO}_{2}^{+}\left(\tilde{B}^{2} \Sigma_{u}^{+}\right), \mathrm{CO}_{2}^{2+}\right.$, $\mathrm{O}^{+}, \mathrm{O}^{2+}, \mathrm{CO}^{+}, \mathrm{C}^{+}, \mathrm{C}^{2+}$, ionization to carbon $\mathrm{K}$-shell, ionization to oxygen $\mathrm{K}$-shell) are also included in the set. Moreover, dissociative attachment of $\mathrm{CO}_{2}$ into $\mathrm{CO}$ and $\mathrm{O}^{-}$is considered.

It should be noted that other sets of electron scattering cross sections in $\mathrm{CO}_{2}$ are available in LXCat. In particular, the ISTLisbon database includes a comprehensive set of cross sections that are optimized for the calculation of swarm transport coefficients from the solution of a two-term homogeneous EBE [28]. However, in this work, for the sake of numerical benchmarking and consistency with the MC approach implemented, the Biagi database has been preferred since it provides a complete set of cross sections to be used for solving the EBE under multi-term expansion or MC calculations. It is worth noting that, at the moment, there are large discrepancies between cross sections for electron kinetics in $\mathrm{CO}_{2}$ of different datasets. Those discrepancies involve particularly the description of electronic excitation and dissociation processes [69]. In this respect, this work also aims to assess the validity of Biagi cross sections for describing electron kinetics in $\mathrm{CO}_{2}$ in $\mathrm{MC}$ (or multi-term) codes.

An example of temporal evolution of the EEDF in $\mathrm{CO}_{2}$ obtained with the Biagi cross sections is shown in figure 1. Calculations were performed with MCF at a constant reduced electric field of $50 \mathrm{Td}$, assuming a fixed gas composition of pure $\mathrm{CO}_{2}$ at $N=7.24 \times 10^{22} \mathrm{~m}^{-3}$ (collapsed in the $\mathrm{CO}_{2}(000)$ level). As regards numerical parameters, the energy domain is discretized with $\Delta \epsilon=0.1 \mathrm{eV}$ and $\epsilon_{\max }=20 \mathrm{eV}$. The simulation is initiated by placing $n_{\mathrm{p}}=10^{5}$ electrons per cell that are used for the calculation of transition probabilities within the time interval $\Delta t=1.8 \times 10^{-9} \mathrm{~s}$. The time resolved EEDF is computed by an iterative application of equation (1) to an initial Boltzmann distribution of electrons at $300 \mathrm{~K}$, until reaching steady-state at around $1.8 \times 10^{-7} \mathrm{~s}$.

It is worth noting that, in the energy range between 1.0 and $5.0 \mathrm{eV}$, steady-state is established after only $5.4 \times 10^{-9}$ $\mathrm{s}$, that is about a factor 10 less than for the lower energies portion $(\epsilon<1.0 \mathrm{eV})$ and the tail of the distribution $(\epsilon>4.0 \mathrm{eV})$. This reflects the differences between energy and momentum

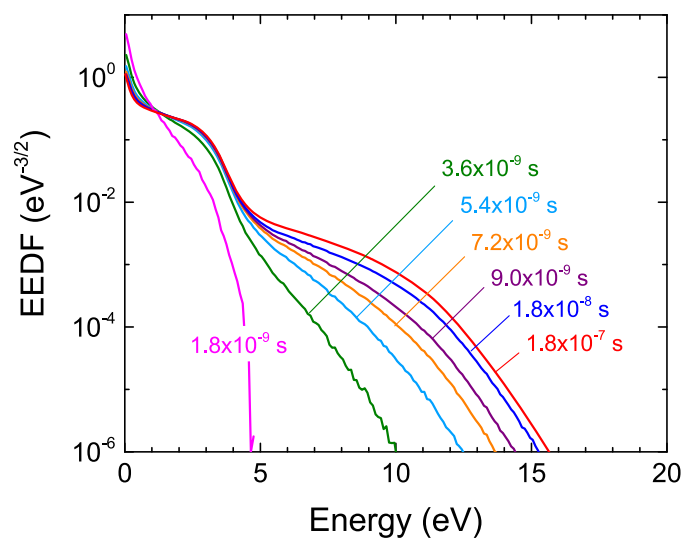

Figure 1. Time evolution of the EEDF in $\mathrm{CO}_{2}$ under a constant reduced electric field of $50 \mathrm{Td}$ and gas number density of $7.24 \times 10^{22} \mathrm{~m}^{-3}$. A time step of $\Delta t=1.8 \times 10^{-9} \mathrm{~s}$ is used in MCF calculations of transition probabilities.

relaxation frequencies in each energy range. When steadystate is reached, a tail appears in the EEDF starting from around $7 \mathrm{eV}$ following a drop in the distribution. This feature is typical of molecular gases with high vibrational cross sections [1]. In fact, those inelastic processes act as a sharp barrier that electrons have to overcome to reach higher energies. In addition to that, as mentioned before, several different inelastic processes involving mainly electron impact dissociation of $\mathrm{CO}_{2}$ are present in the energy region between 6.5 and $8.75 \mathrm{eV}$. The CPU time for MCF simulations for these conditions, including calculations of transition probabilities and time resolved EEDFs, is about 1 min with an Intel Fortran compiler on an Intel Xeon CPU E5-2637 v3 @ 2.50 GHz processor. In MCF, most of the CPU time is spent on calculation of transition probabilities, whereas the solution of the deterministic problem is typically very fast (of the order of a few milliseconds, depending on the size of the matrix). In total, the CPU time from MCF calculations is about a factor 3 higher than the one from steady-state solutions with the multi-term solver MultiBolt [35] with similar accuracy in rate coefficient calculations, as shown in more details later in this section.

In the following subsection emphasis is given to benchmark calculations of Legendre polynomial coefficients and rate coefficients from MCF and Boltzmann solvers using the two-term (BOLSIG+ [23]) and ten-term (MultiBolt [35]) expansion in Legendre polynomials. The benchmark is limited to steady-state conditions. Two different case studies are considered, corresponding to moderate and high $E / N$. For the sake of codes benchmarking, the following assumptions have been included:

- An invariant chemical composition of pure $\mathrm{CO}_{2}$ as a background gas is considered. In this gas, the population is collapsed in the $\mathrm{CO}_{2}(000)$ state. In this way, $\mathrm{CO}_{2}$ is modelled as an ideal gas, where the population of internal states at a finite gas temperature is neglected. Even if the first vibrational levels of the ground state can be largely populated already at room temperature, this strong assumption is made for the sake of numerical benchmarking with other solvers (i.e. MultiBolt), that 
do not include options for population of excited states. Extension of the model by considering finite gas temperature is discussed in section 3.2. Moreover, the effect of inclusion of population of internal energy levels is shown in section 3.3.

- The gas is weakly ionized, such that the effect of selfcollisions of electrons can be neglected (in the spirit of the linear Boltzmann equation).

- An homogeneous and time-independent electric field is considered, such that non-local effects of electron kinetics can be neglected.

- All elastic and inelastic scattering collisions are treated as isotropic. Although anisotropic scattering may have important effects on electron transport at high $E / N$, detailed collision dynamics is not included in the Boltzmann solvers used for benchmarking.

\subsection{Cold gas approximation}

The MCF method is applied to the case of electrons in $\mathrm{CO}_{2}$ by calculating the EVDF under the cold gas approximation, in steady-state and homogeneous conditions. Results of MCF are benchmarked in the range $E / N=20-1000 \mathrm{Td}$ against the codes BOLSIG + [23] and MultiBolt [35] for the calculation of the first three Legendre polynomial coefficients. At low $E / N$ values, benchmarking with the two-term solver is very good as expected (not shown). The validity of the widely used twoterm approximation is verified for the calculation of rate coefficients of inelastic processes such as vibrational excitation and $\mathrm{CO}_{2}$ dissociation.

3.1.1. Moderate reduced electric fields: $E / N=20-100 T d$. MCF calculations are performed with the following numerical parameters: $\Delta \epsilon=0.1 \mathrm{eV}, \epsilon_{\max }=20 \mathrm{eV}, n_{\cos \theta}=50$ and $n_{\mathrm{p}}=10^{4}$. In particular, a discretization in $\cos \theta$ is needed for computing higher order Legendre polynomial coefficients, whereas setting $n_{\cos \theta}=1$ is sufficient for the representation of the isotropic part of the distribution. Default numerical parameters are used in the two-term Boltzmann solver BOLSIG+ (precision $=10^{-10}$, convergence $=10^{-5}$, number of iterations $=2000$ and 100 energy intervals). The MultiBolt solver was used in the hydrodynamic regime, under a ten-term expansion in Legendre polynomials of the velocity distribution function with default parameters (convergence error in mean energy $=10^{-6}$, maximum number of iterations $=2000$, energy remap $=1$, number of energy intervals $=1000$ ). In figure 2, it can be noticed that the EEDFs obtained with MCF and MultiBolt present significant deviations with respect to the ones calculated with BOLSIG + . This is particularly visible in the tail of the distributions, for $E / N<80 \mathrm{Td}$.

The discrepancy in results from the two-term approach is mainly due to the presence of a pronounced Ramsauer minimum in the elastic momentum transfer cross sections in the energy range of interest. Due to the high number of vibrational excitation processes, the collision frequency of inelastic processes reaches values comparable with the one of elastic processes. Hence, the small energy variation assumption implicit in the two-term approach is not valid [31]. Calculations of $f_{1}$

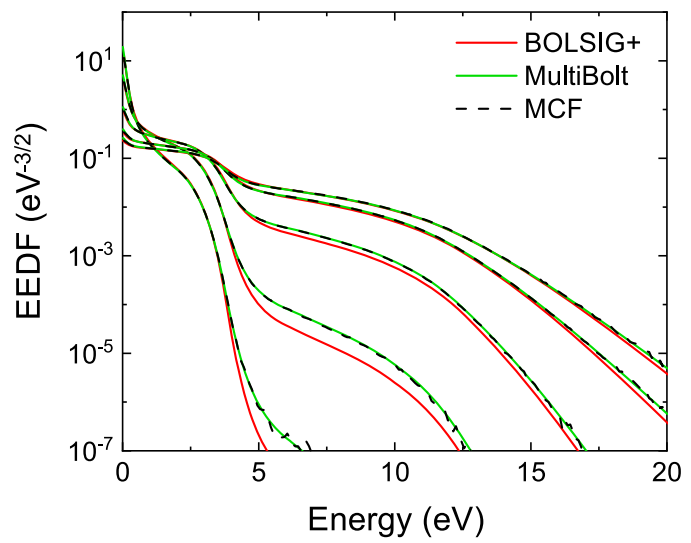

Figure 2. Zeroth-order Legendre polynomial coefficient calculated with MCF, BOLSIG + (two-term solver) and MultiBolt (ten-term solver) in $\mathrm{CO}_{2}$ at different values of constant reduced electric field. From left to right: 20, 30, 50, 80 and 100 Td.

and $f_{2}$ (figure 3 ), for the same values of $E / N$, show that the two Legendre polynomial coefficients have absolute values similar to the corresponding EEDFs. The strong coupling between the first three coefficients of the expansion is another explanation for the discrepancies in results from the two-term approach. Moreover, comparing MCF results of figure 2 with figures 3(a) and (b), it is possible to notice that higher order Legendre polynomial coefficients present slightly higher stochastic fluctuations in the tail of the distribution. This is due to the fact that, in MC methods, Legendre polynomial coefficients are calculated from the $n$-particle distribution function, where each of the $n$ simulated electrons contributes as a term weighted by the respective Legendre polynomial [70]. For this reason, anisotropic components of the distribution suffer from lower counting statistics compared to the isotropic one.

Total rate coefficients for the vibrational excitation of $\mathrm{CO}_{2}(000)$ to $\mathrm{CO}_{2}(010), \mathrm{CO}_{2}(020)$ and $\mathrm{CO}_{2}(110+030)$ and for electron impact dissociation are calculated with $\mathrm{MCF}$ and BOLSIG + and compared to results from MultiBolt. Results are shown in figures 4(a) and (b), respectively. Since vibrational excitation processes have a low energy threshold, deviation in the rate coefficients of the two-term solver $\mathrm{BOLSIG}+$ is within 5\%, whereas MCF results are within $1 \%$ compared with MultiBolt (figure 4(a)). Rate coefficients of inelastic processes that have threshold near the tail of the distribution are more sensitive to departures of the EEDF from the multi-term solution. This is shown in figure 4(b) for the electron impact dissociation processes. In this case, the relative difference with multi-term calculations can exceed $70 \%$, whereas MCF deviations are within 3\%. Discrepancies between MCF results and MultiBolt calculations are mainly due to the different energy grids in the two codes and to the presence of small stochastic fluctuations in the EEDFs from MCF, that can be reduced by increasing the number of simulated particles. As regards rate coefficients of inelastic processes, similar results were obtained by Braglia and co-authors [48] when comparing a conventional MC with a two-term solver. In particular, they pointed out the inadequacy of the two-term approach for calculation of certain rate coefficients, like electronic excitations, in a range of reduced electric fields from 10 to 100 

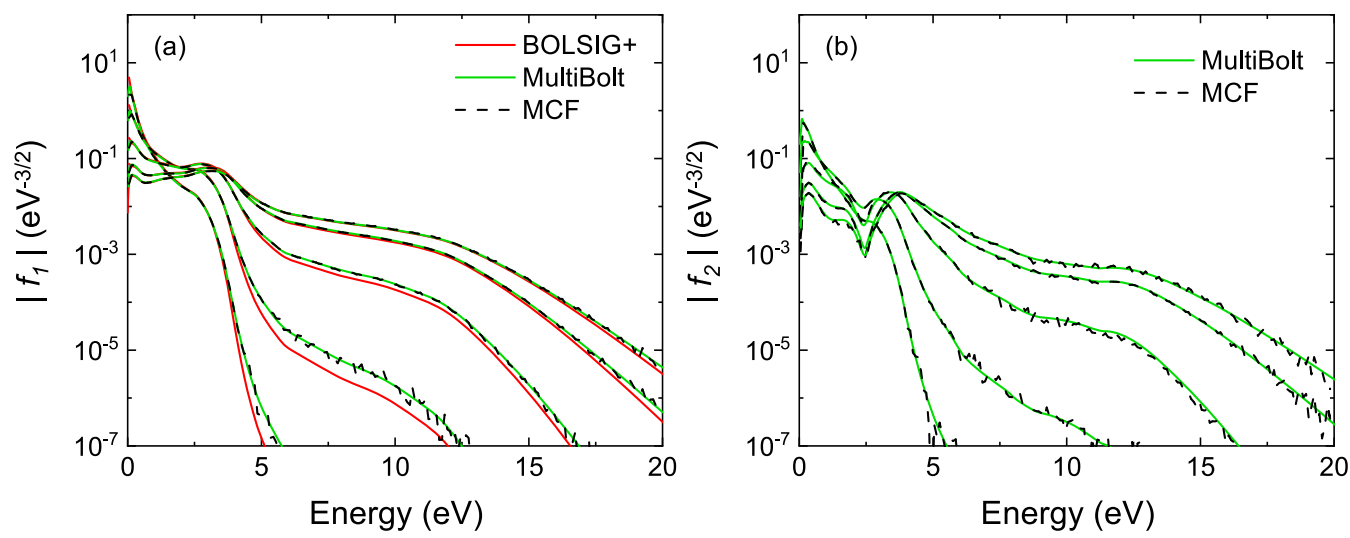

Figure 3. (a) First order and (b) second order Legendre polynomial coefficients calculated with MCF, BOLSIG+ (two-term solver) and MultiBolt (ten-term solver) at different constant reduced electric fields in $\mathrm{CO}_{2}$. From left to right: 20, 30, 50, 80 and $100 \mathrm{Td}$. Calculations of $f_{2}$ are compared to results from MultiBolt only, since BOLSIG+ solutions are truncated at the first order.
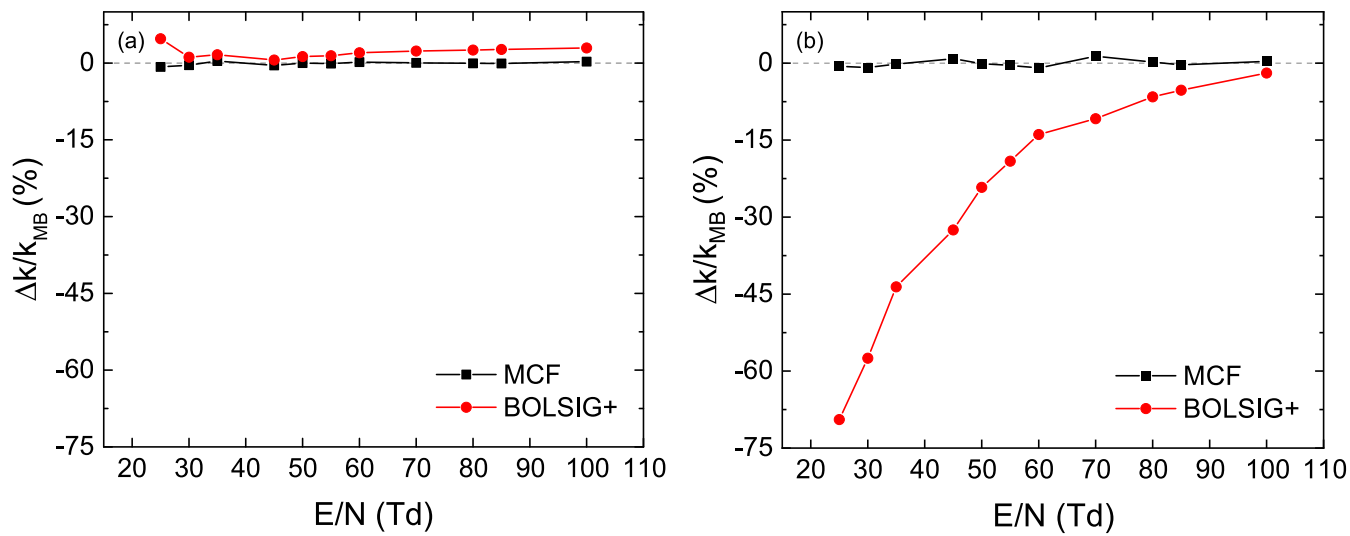

Figure 4. Relative difference (in percent) of total rate coefficients in $\mathrm{CO}_{2}$ of (a) vibrational excitation $\mathrm{CO}_{2}(000) \rightarrow \mathrm{CO}_{2}(010)$, $\mathrm{CO}_{2}(020)$ and $\mathrm{CO}_{2}(110+030)$ and (b) electron impact dissociation to $\mathrm{CO}$ and $\mathrm{O}$ calculated from MCF and BOLSIG+ (two-term solver) with respect to MultiBolt calculations (ten-term solver), at different reduced electric fields.

Td. In their work, using a different set of electron impact cross sections, the error from a two-term solution was up to $40 \%$ for the rate coefficient of electronic excitation with threshold $7.0 \mathrm{eV}$ at $E / N=40 \mathrm{Td}$.

3.1.2. High reduced electric fields: $E / N=400-1000 T d$. In this section we study electron transport at high $E / N$. As already mentioned, for benchmarking purposes, collision dynamics of elastic and inelastic processes is assumed to be isotropic in velocity space. This choice is dictated by the fact that none of the other two Boltzmann solvers include options for anisotropic scattering. However, anisotropic collision dynamics is particularly important at high $E / N$, since it can have an impact on the EVDF shape and on the average quantities calculated from it [71]. Moreover, equal energy sharing between the primary and secondary electron is assumed in ionization events. In this case, a more correct treatment of energy sharing should include singly differential cross sections [63, 72]. As an alternative, when the energy of the incident electron is just above the ionization threshold, a good approximation in MC models is to distribute randomly the energy remaining, after subtracting the ionization energy, between the primary and secondary electron with a uniform distribution. Future investigations will focus on more advanced treatment of collision dynamics at high $E / N$. In this case, the numerical parameters used in MCF are: $\Delta \epsilon=0.3 \mathrm{eV}$, $\epsilon_{\max }=120 \mathrm{eV}, n_{\cos \theta}=50$ and $n_{\mathrm{p}}=10^{4}$. The same numerical parameters as in previous case studies are used for BOLSIG+ and MultiBolt. Results for EEDFs calculated at 400, 600 and $1000 \mathrm{Td}$ are shown in figure 5.

Large deviations from a two-term solution can be noticed in the EEDF calculations (figure 5). This is mostly due to the presence of electronic excitation and ionization processes that are dominant for energies above $30 \mathrm{eV}$. Moreover, at such high energies, the electron motion is driven by the presence of strong electric fields that set a preferential direction in velocity space. In those conditions, the first and second Legendre polynomial coefficients for energies above $30 \mathrm{eV}$ are even higher than the corresponding isotropic component, as shown in figure 6. The discrepancies between two-term solutions and the ones obtained with the other methods affect the calculation of flux transport coefficients, such as reduced mobility and components of the diffusion tensor (not shown).

However, such deviations for distributions calculated with the two-term approximation do not impact considerably 


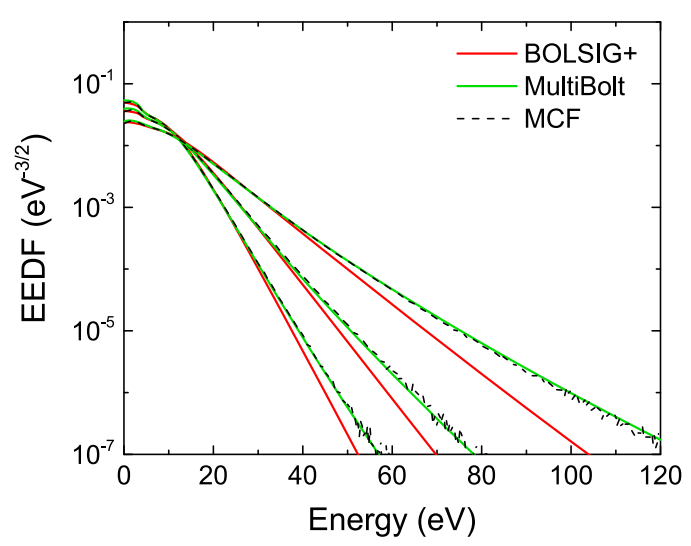

Figure 5. Zeroth order Legendre polynomial coefficient calculated with MCF, BOLSIG+ (two-term solver) and MultiBolt (ten-term solver) in $\mathrm{CO}_{2}$ at different values of constant reduced electric field. From left to right: 400, 600 and $1000 \mathrm{Td}$.

the calculation of rate coefficients of inelastic processes. An example is shown in figure 7 for the same inelastic processes of excitation to bending mode levels and electron impact dissociation as in the previous section. It can be noted that BOLSIG + calculations present relative errors within 5\%, whereas MCF results are within $2 \%$, mainly because of effects of the numerical discretization of the energy domain.

\subsection{Finite gas temperature of the background gas}

The MCF model described in [54] has been upgraded to consider effects of finite gas temperature of the background gas on the EVDF. In this section, the focus is on low values of reduced electric fields, such that the mean electron energy is comparable with the thermal energy of the background gas. In this context, MCF results are compared with $\mathrm{BOLSIG}+$, that includes a Boltzmann-Davydov term in the elastic operator. It is important to notice that, in this subsection, the population of vibrational and rotational levels of $\mathrm{CO}_{2}$ ground state is neglected. In this way, the background gas is treated as an ideal one, where the gas temperature has only effects in energy exchanges between neutral molecules and electrons in elastic collisions. Reduced electric fields between 0.5 and $1.5 \mathrm{Td}$ are considered, together with gas temperatures between 300 and $1000 \mathrm{~K}$. The partial dissociation of the gas which occurs at the highest temperature has also been neglected. As regards the MCF code, the test Particle MC method described in [57] has been implemented. The scattering is treated in the centre-ofmass frame and requires cross sections in the same reference frame. Conversion of cross sections from the laboratory to the centre-of-mass frame depends on the thermal energy of the target particles [73]. In this case, however, the difference between cross sections in the two reference frames is negligible, due to the low electron-to-molecule mass ratio. As described in [57], in case of elastic collisions, the relative velocity vector is randomly rotated isotropically in velocity space. In case of inelastic collisions, the relative velocity is updated taking into account the energy loss involved in the process.

MCF calculations are performed with the following numerical parameters: $\Delta \epsilon=0.002 \mathrm{eV}, \epsilon_{\max }=0.25 \mathrm{eV}, n_{\cos \theta}=1$ and $n_{\mathrm{p}}=10^{4}$. Default numerical parameters are used in the two-term Boltzmann solver BOLSIG + (precision $=10^{-10}$, convergence $=10^{-5}$, number of iterations $=2000$ and 100 energy intervals). Since MultiBolt does not have an option for the description of finite gas temperature effects, it was not used for benchmarking in this section. An example of EEDFs calculated at $T_{\text {gas }}=0$ (cold gas), 500 and $1000 \mathrm{~K}$ is shown in figure 8 (a) for a constant $E / N$ of $0.5 \mathrm{Td}$. As expected, the finite gas temperature enhances the tail of the EEDF due to the transfer of energy from neutral target particles to electrons. The first Legendre polynomial coefficients are shown in figure 8(b), where it can be noted that the effect of the gas temperature is similar to the one for $f_{0}$.

It should be considered that, due to the necessity of sampling velocity components of the colliding partners, this MCF extension is more computationally expensive. In fact, while EEDF calculations with a frozen background gas require about 1 min only, having a finite $T_{\text {gas }}$ leads to calculations that are about 3 times longer, mainly because of the generation of additional random numbers for sampling the velocity components of the molecules. An improvement in MCF computational performances can be achieved, for example, by employing high performance computing techniques, such as parallelization or multi-threading.

Effects of finite gas temperature of the background gas can also be appreciated by comparing rate coefficients of inelastic processes at low $E / N$. In figure 9, total rate coefficients for the vibrational excitation processes to bending mode levels $\left(\mathrm{CO}_{2}(000) \rightarrow \mathrm{CO}_{2}(010), \mathrm{CO}_{2}(020)\right.$ and $\left.\mathrm{CO}_{2}(110+030)\right)$ are calculated at different $T_{\text {gas }}$ from 300 to $1000 \mathrm{~K}$, in a range of $E / N$ from 0.5 to $1.5 \mathrm{Td}$ and compared with results of MCF obtained with the cold gas approximation. The relative difference of MCF results with respect to cold gas calculations at $T_{\text {gas }}=0 \mathrm{~K}$ increases at lower values of $E / N$ and exceeds $80 \%$ for $T_{\mathrm{gas}}=1000 \mathrm{~K}$ at $0.5 \mathrm{Td}$.

\subsection{Effect of gas kinetics and inclusion of population of excited states}

Until now, for numerical benchmarking purposes, the $\mathrm{CO}_{2}$ system has been modelled as an ideal gas, where the population of excited states has been neglected by assuming that vibrational states of the ground electronic state are collapsed into the $\mathrm{CO}_{2}(000)$ state. In particular, for the $\mathrm{CO}_{2}$ case, this assumption breaks down already at room temperature, where the first vibrationally excited levels (e.g. $\mathrm{CO}_{2}(010)$ ) can be significantly populated [28]. Here we compare results from the previous section with calculations that include population of excited states. In this section, electron transport at low $E / N$ is studied, where the population of the first six low-energy vibrational levels of the $\mathrm{CO}_{2}$ ground electronic state has been considered. In particular, together with the ground vibrational state $\mathrm{CO}_{2}(000)$, we consider:

- The first vibrational level of the asymmetric stretching mode $\left(\mathrm{CO}_{2}(001)\right)$.

- The first vibrational level of the symmetric stretching mode $\left(\mathrm{CO}_{2}(100)\right)$. 

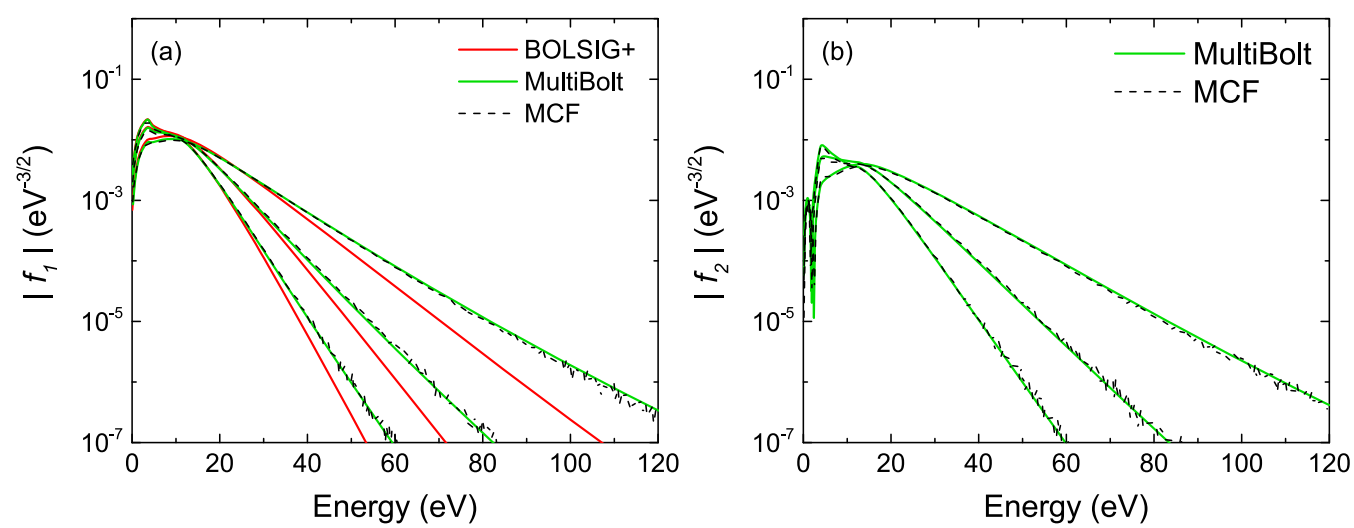

Figure 6. (a) First order and (b) second order Legendre polynomial coefficients calculated from MCF, BOLSIG+ (two-term solver) and MultiBolt (ten-term solver) at different constant reduced electric fields in $\mathrm{CO}_{2}$. From left to right: 400, 600 and $1000 \mathrm{Td}$. Calculation of $f_{2}$ are compared with MultiBolt only, since BOLSIG+ solutions are truncated at the first order.
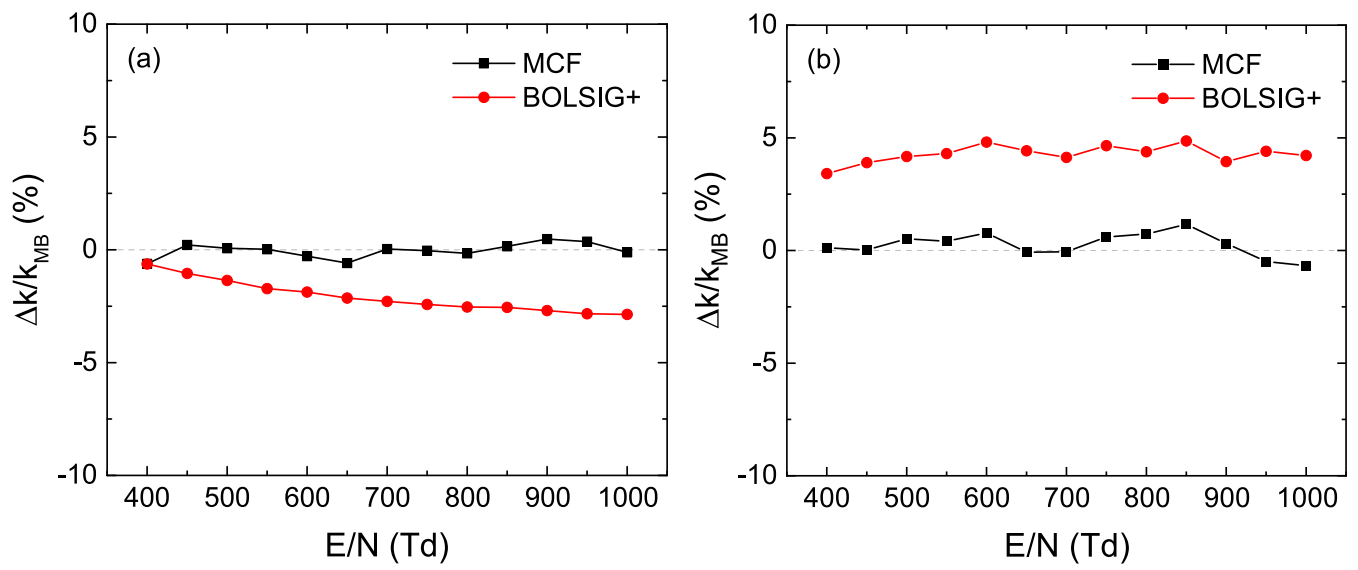

Figure 7. Relative difference (in percent) of total rate coefficients in $\mathrm{CO}_{2}$ of (a) vibrational excitation $\mathrm{CO}_{2}(000) \rightarrow \mathrm{CO}_{2}(010), \mathrm{CO}_{2}(020)$ and $\mathrm{CO}_{2}(110+030)$ and (b) electron impact dissociation to $\mathrm{CO}$ and $\mathrm{O}$ calculated from MCF and BOLSIG+ (two-term solver) with respect to MultiBolt calculations (ten-term solver), at different reduced electric fields.

- The first three effective vibrational levels of the bending mode (i.e. $\mathrm{CO}_{2}(010), \mathrm{CO}_{2}(020)$ and $\mathrm{CO}_{2}(030+110)$ with degeneracy equal to 2, 3 and 6, respectively. The last one presents Fermi resonance between symmetric stretching and bending mode levels).

In this way, the $\mathrm{CO}_{2}$ molecule is assumed to be a truncated system composed by six vibrational levels of the ground electronic state, that can be populated by electron impact vibrational excitation and de-populated through superelastic collisions. Cross sections for superelastic processes are calculated by assuming micro-reversibility, with the Klein-Rosseland formula [15]. In order to correctly describe the $\mathrm{CO}_{2}$ molecule using an MC method and the Biagi database and to compare results with different methods, the set of cross sections requires a specific treatment. In this respect, it is important to notice that the elastic momentum transfer cross section of the Biagi database takes into account the contribution of the ground electronic state, rotational states and vibrational bending mode in thermal equilibrium at a fixed $T_{0}=293.15 \mathrm{~K}$. Since we want to populate the aforementioned vibrational states according to a Boltzmann distribution at a generic vibrational temperature
$T_{\text {vibr }}$, a change in the elastic momentum transfer cross sections for $T_{\text {vibr }} \neq T_{0}$ has to be taken into account. In this work, the following formula is used, according to the Magboltz source code v11.3 [47]:

$$
\begin{aligned}
\left(\sigma_{\mathrm{el}}(\epsilon)\right)_{T_{\mathrm{vibr}}}= & \frac{1-\xi_{\text {bend }}}{\alpha_{\text {ground }}}\left[\left(\sigma_{\mathrm{el}}(\epsilon)\right)_{T_{0}}-\alpha_{\text {bend }} \sigma_{\text {bend }}(\epsilon)\right] \\
& +\xi_{\text {bend }} \sigma_{\text {bend }}(\epsilon)
\end{aligned}
$$

where $\left(\sigma_{\mathrm{el}}(\epsilon)\right)_{T_{\mathrm{vibr}}},\left(\sigma_{\mathrm{el}}(\epsilon)\right)_{T_{0}}$ and $\sigma_{\text {bend }}(\epsilon)$ are the cross section of elastic momentum transfer calculated at $T_{\text {vibr }}$, the cross section provided in the Biagi database of LXCat [66] (at $T_{0}$ ) and the cross section of elastic momentum transfer of the bending mode (that is tabulated separately in LXCat [66]), respectively. The term $\xi_{\text {bend }}$ is the fractional population of the bending mode levels (i.e. $\mathrm{CO}_{2}(010), \mathrm{CO}_{2}(020)$ and $\mathrm{CO}_{2}(030+110)$ ) calculated at $T_{\text {vibr }}$, whereas $\alpha_{\text {bend }}$ is the fractional population of the bending mode levels at $T_{0}$ and $\alpha_{\text {ground }}$ is the fractional population of the other vibrational levels of the ground electronic state (i.e. $\alpha_{\text {ground }}=1-\alpha_{\text {bend }}$ ). Note that, in case $T_{\text {vibr }}=T_{0}$, the calculated elastic momentum transfer cross 

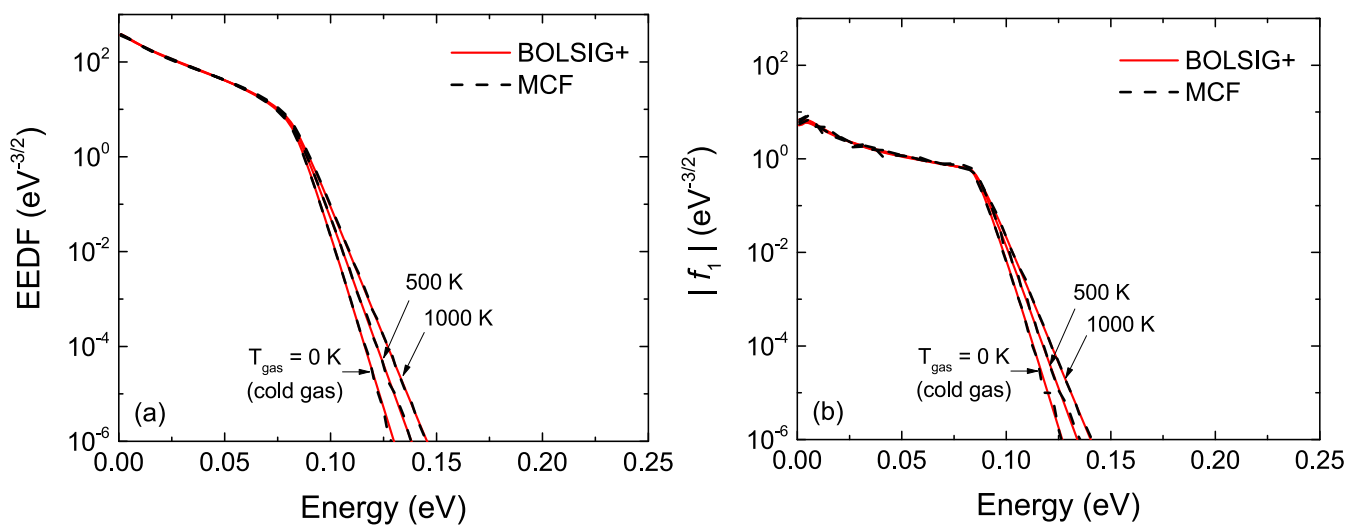

Figure 8. (a) Zeroth and (b) first order Legendre polynomial coefficients calculated with MCF and BOLSIG+ at 0.5 Td for different gas temperatures in $\mathrm{CO}_{2}$. Calculation at $T_{\text {gas }}=0$ corresponds to frozen background gas (cold gas), whereas results at $T_{\text {gas }}=500$ or $1000 \mathrm{~K}$ include finite gas temperatures.

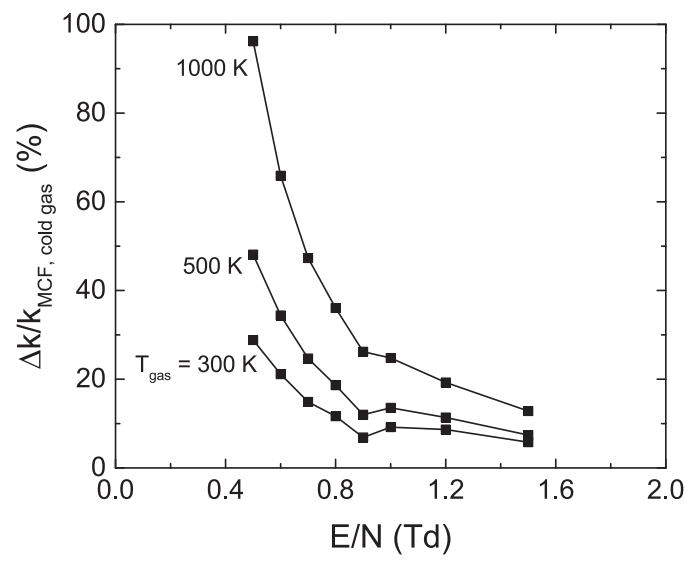

Figure 9. Relative difference (in percent) of $\mathrm{MCF}$ total rate coefficients for vibrational excitations $\left(\mathrm{CO}_{2}(000) \rightarrow \mathrm{CO}_{2}(010)\right.$, $\mathrm{CO}_{2}(020)$ and $\left.\mathrm{CO}_{2}(110+030)\right)$ obtained at $T_{\mathrm{gas}}=300,500$ and $1000 \mathrm{~K}$, with respect to MCF results obtained under cold gas assumption, as a function of the reduced electric field.

section is equal to the one reported in the Biagi database of LXCat (i.e. $\left.\left(\sigma_{\mathrm{el}}(\epsilon)\right)_{T_{\mathrm{vibr}}}=\left(\sigma_{\mathrm{el}}(\epsilon)\right)_{T_{0}}\right)$. The elastic momentum transfer cross section from the Biagi database $\left(\left(\sigma_{\mathrm{el}}(\epsilon)\right)_{T_{0}}\right)$ is reported in figure 10, together with the one calculated at $T_{\text {vibr }}=1000 \mathrm{~K}\left(\left(\sigma_{\text {el }}(\epsilon)\right)_{T_{\text {vibr }}}\right)$ and the one of the bending mode levels $\left(\sigma_{\text {bend }}(\epsilon)\right)$. In the energy range between 0.1 and $5.0 \mathrm{eV}$, the cross section obtained at $T_{\text {vibr }}=1000 \mathrm{~K}$ is higher than the corresponding one at $T_{0}=293.15 \mathrm{~K}$. This is due to the fact that, at higher vibrational temperatures, the fractional population of bending mode levels is higher than the one at room temperature, thus the calculated cross section is closer to the one for bending mode levels. The increase in the elastic momentum transfer cross section in $\mathrm{CO}_{2}$ with increasing population of vibrational states has been investigated experimentally by Buckman and co-authors [74] and inconsistencies arising by neglecting this effect have been noted by Haddad and Elford [75], for the calculations of electron drift velocities in $\mathrm{CO}_{2}$ at different gas temperatures.

In order to be consistent with the elastic momentum transfer cross section used in the present work, rotational excitation

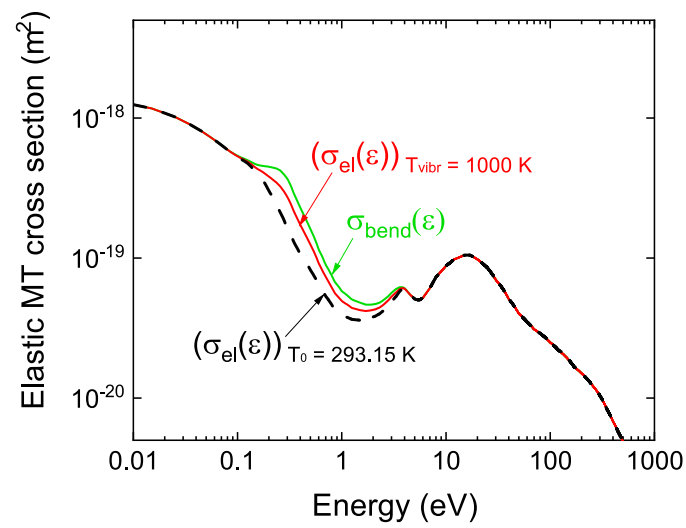

Figure 10. Elastic momentum transfer cross sections of $\mathrm{CO}_{2}$ considering the contribution of the bending mode levels $\left(\sigma_{\text {bend }}(\epsilon)\right)$, for electron impact with molecules in the ground electronic state at $T_{\text {vibr }}=1000 \mathrm{~K}\left(\left(\sigma_{\mathrm{el}}(\epsilon)\right)_{T_{\text {vibr }}}\right)$ and for electron impact with molecules in the ground electronic state provided in the Biagi database of LXCat $\left(\left(\sigma_{\mathrm{el}}(\epsilon)\right)_{T_{0}}\right)$ for thermal equilibrium at $T_{0}=293.15 \mathrm{~K}$.

and de-excitation processes must be taken into account. At present, the Biagi database of LXCat does not include rotational cross sections. However, those processes are included in the Magboltz source code v11.3 [47]. Rotational excitation and de-excitation cross sections were calculated considering electric quadrupole transitions that are characterized by a difference between initial and final rotational quantum numbers of $\Delta J= \pm 2$ [76]. Cross sections in [76] are valid for low energy electrons, since the principal contribution comes from large distances of the incoming electrons from the molecules. For energies greater than about $6.0 \mathrm{eV}$, an artificial Born decay of $1 / \epsilon$ in the cross sections has been introduced, as in the Magboltz source code [47]. In the present work, 31 rotational states of the $\mathrm{CO}_{2}$ ground state (i.e. even values of $J$ from 0 to 60 ) are considered and they are populated according to a Boltzmann distribution at temperature $T_{\text {rot }} \neq T_{0}$. Using the formulas for cross sections for rotational excitations $\sigma_{J, J+2}(\epsilon)$ and de-excitations $\sigma_{J, J-2}(\epsilon)$, it is possible to subtract their contribution from the elastic momentum transfer cross section $\left(\sigma_{\mathrm{el}}(\epsilon)\right)_{T_{\text {vibr }}}($ defined in equation (5)) as 


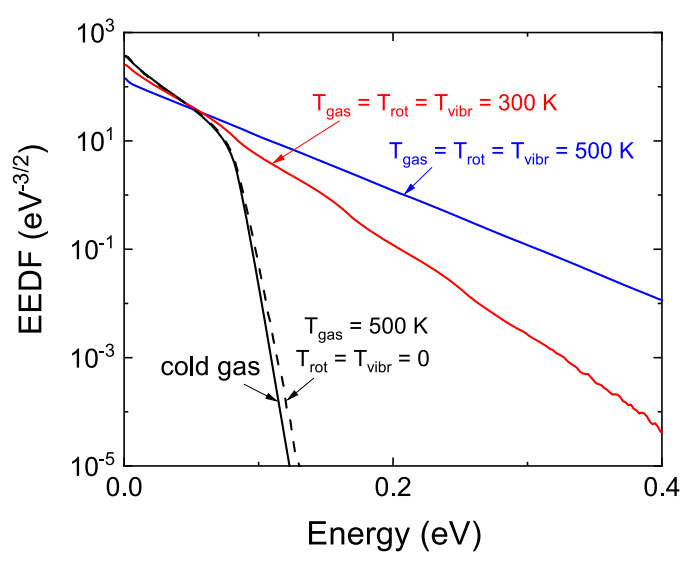

Figure 11. EEDFs in $\mathrm{CO}_{2}$ at $0.5 \mathrm{Td}$ considering cold gas assumption with frozen background and neglecting population of excited states; hot background of $\mathrm{CO}_{2}$ molecules at $T_{\text {gas }}=500 \mathrm{~K}$ neglecting population of excited states $\left(T_{\text {rot }}=T_{\text {vibr }}=0\right)$ and hot background at $T_{\text {gas }}=300 \mathrm{~K}(500 \mathrm{~K})$ and Boltzmann population of rotationally and vibrationally excited states at $T_{\text {rot }}=T_{\text {vibr }}=300 \mathrm{~K}(500 \mathrm{~K})$.

$$
\begin{aligned}
\left(\sigma_{\mathrm{el}}(\epsilon)\right)_{T_{\mathrm{vibr}}, T_{\mathrm{rot}}}= & \left(\sigma_{\mathrm{el}}(\epsilon)\right)_{T_{\mathrm{vibr}}}-\sum_{J}\left[\xi_{J}^{\mathrm{exc}} \sigma_{J, J+2}(\epsilon)\right. \\
& \left.+\xi_{J}^{\mathrm{de}-\mathrm{exc}} \sigma_{J, J-2}(\epsilon)\right]
\end{aligned}
$$

where $\left(\sigma_{\text {el }}(\epsilon)\right)_{T_{\text {vibr }}, T_{\text {rot }}}$ is the elastic momentum transfer cross section obtained by considering the contribution of the population of bending mode levels at $T_{\text {vibr }}$ and by subtracting the contribution of the inelastic rotational cross sections. The terms $\xi_{J}^{\mathrm{exc}}$ and $\xi_{J}^{\mathrm{de}-\mathrm{exc}}$ are the fractional populations at $T_{\text {rot }}$ of the rotational states considered in rotational excitations and de-excitations, respectively. We note that, for the present conditions, effects of rotational excitations and de-excitations are negligible compared to vibrational energy losses, in agreement with [77].

In order to investigate the effect of population of energy levels of the $\mathrm{CO}_{2}$ ground electronic state on EEDF calculations, MCF simulations have been performed. Results are shown in figure 11 for a constant $E / N=0.5 \mathrm{Td}$. The same numerical parameters as in the previous subsection were used. In particular, figure 11 shows a comparison of the EEDF obtained under cold gas assumption (in which the gas temperature, rotational and vibrational temperature are set to zero) with the one obtained by setting higher temperatures. First of all, as noticed in the previous subsection, by setting $T_{\text {gas }}=500 \mathrm{~K}\left(\neq T_{0}\right)$ and $T_{\text {rot }}=T_{\text {vibr }}=0$, effects of gas kinetics are included by considering the energy exchange with the hot target molecules. However, this approach neglects the contribution of excited states of the ground electronic state. Moreover, we notice that this approach is inconsistent with the use of the elastic momentum transfer cross section from the Biagi database, that takes into account ground electronic state, bending mode and rotational contribution in thermal equilibrium at 293.15 K. In figure 11, results of EEDFs obtained at $T_{\text {gas }}=T_{\text {rot }}=$ $T_{\text {vibr }}=300 \mathrm{~K}$ and $T_{\text {gas }}=T_{\text {rot }}=T_{\text {vibr }}=500 \mathrm{~K}$ are also shown, in which vibrational and rotational states are populated according to a Boltzmann distribution at $T_{\text {vibr }}$ and $T_{\text {rot }}$, respectively.

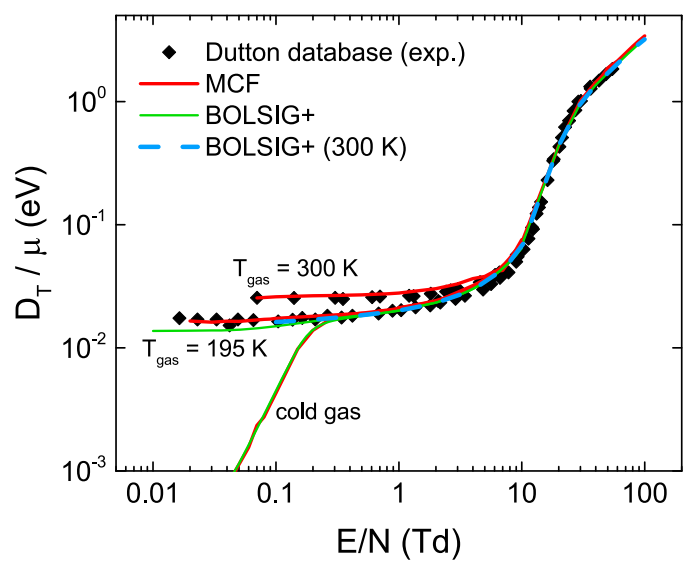

Figure 12. Measured characteristic energies in $\mathrm{CO}_{2}$ [78] and calculated values from BOLSIG + [23] and MCF, considering cold gas approximation or finite gas temperatures.

As it can be noticed, the EEDFs obtained in these conditions present a higher tail. This is mostly due to the presence of superelastic vibrational collisions, in conditions where the electron temperature is comparable with $T_{\text {vibr }}$. A secondary cause of differences between calculations at 300 and $500 \mathrm{~K}$ is due to the fact that higher temperatures lead to an increase of the elastic momentum transfer cross section in the energy range of interest (from equations (5) and (6)), thus leading to a higher elastic collision frequency with respect to the inelastic one.

\subsection{Comparison with experimental results}

The characteristic energy (i.e. the ratio between transversal diffusion coefficient and mobility) from BOLSIG + [23] and $\mathrm{MCF}$, using the Biagi dataset of cross sections [66] is compared. Results are shown in figure 12, together with experimental values from the Dutton database of LXCat [78] at $T_{\text {gas }}=195$ and $300 \mathrm{~K}$. As expected, BOLSIG + and MCF results with a frozen background (cold gas) deviate from the experimental values for $E / N \leqslant 0.2 \mathrm{Td}$, where the mean electron energy becomes comparable with the thermal energy of the background gas. Moreover, BOLSIG+ results including a Davydov-Boltzmann correction at $300 \mathrm{~K}$ deviate from experimental values at the same $T_{\text {gas }}$ and $E / N<5 \mathrm{eV}$, as well. As opposed to BOLSIG + calculations, in MCF the elastic momentum transfer cross section is modified using equations (5) and (6), that consider population of the vibrational and rotational states of the ground electronic state, according to a Boltzmann distribution at $T_{\text {gas }}$, as explained in the previous subsection. Moreover, superelastic collisions are taken into account. With this treatment, experimental values for the characteristic energy are reproduced by MCF with an error within 3\%. With this comparison, the importance of considering an accurate description of excited states at low $E / N$, together with a thermal background, is emphasized. The inclusion of those states has an effect on the EEDF through superelastic collisions and should be consistent with the choice of the elastic momentum transfer cross section used in the calculations. 


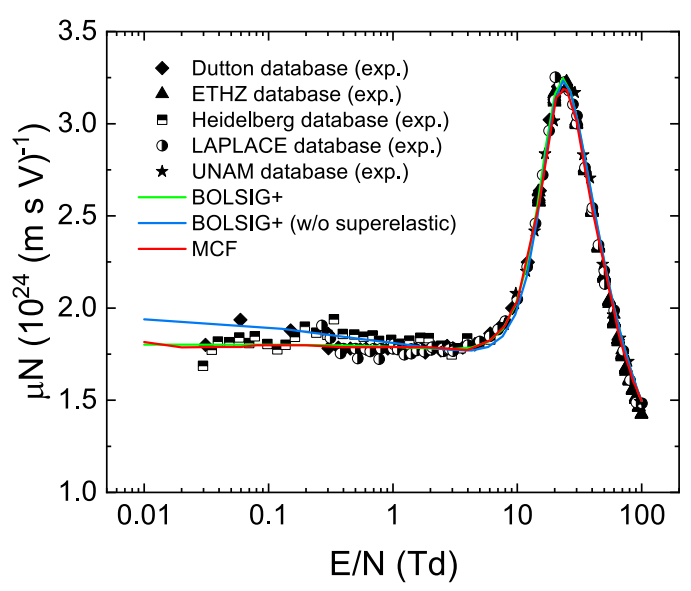

Figure 13. Measured reduced mobilities in $\mathrm{CO}_{2}$ [78-82] and calculated values from MCF and BOLSIG+ [23] at $300 \mathrm{~K}$.

MCF and BOLSIG + [23] calculations of reduced mobilities $(\mu N)$ using Biagi cross sections set are compared with experimental measurements from LXCat databases [78-82]. Results are shown in figure 13 for $0.01<E / N<100 \mathrm{Td}$ at $300 \mathrm{~K}$. Results from MCF and BOLSIG+ show an agreement with experimental data within $2 \%$. A similar agreement, between measurements and calculations of bulk drift velocities, is found by Vass and co-authors [40], using the IST-Lisbon cross sections set [28] in MC and multi-term solvers. However, it is important to notice that there are fundamental differences between the Biagi and the IST-Lisbon datasets. In particular, on the one hand, the Biagi elastic momentum transfer cross section and total cross section (given by the sum of elastic and inelastic contributions) are in good agreement with experimental measurements $[83,84]$, within $3 \%$. On the other hand, the IST-Lisbon elastic momentum transfer cross section is calculated from an effective cross section, by subtraction of the inelastic contributions (weighted by the fractional population of each state) [28]. At $300 \mathrm{~K}$, differences are found between the two datasets, leading to an IST-Lisbon elastic momentum transfer cross section higher than the Biagi one for energies above $3 \mathrm{eV}$. Another important difference between the two datasets lies in the description of the inelastic processes with thresholds above $6 \mathrm{eV}$. In fact, the Biagi set includes several cross sections for dipole-allowed and triplet excitations, as mentioned in section 3, whereas the IST-Lisbon set includes two different processes with threshold of 7 and $10.5 \mathrm{eV}$, as in the Phelps database [85]. Further work is needed to understand the differences between those cross sections sets and their impact on calculations of other swarm parameters, such as the bulk longitudinal diffusion coefficient multiplied by the gas number density $D_{\mathrm{L}} N$, that presents large deviations between experimental measurements and calculations for $E / N$ greater than $100 \mathrm{Td}$ using the currently available cross sections sets [40].

In addition, Biagi cross sections are optimized taking into account superelastic collisions from vibrational and rotational states of the ground electronic state. The importance of those collisions for accurate calculations of swarm data is shown in figure 13, where BOLSIG + calculations of $\mu \mathrm{N}$ are also presented without superelastic process. In particular, for $0.01<E / N<1 \mathrm{Td}$, the difference between BOLSIG+ results obtained by neglecting contributions of superelastic processes and MCF, that intrinsically includes them, is up to $7 \%$. A similar effect of the superelastic collisions on the EVDF and on electron swarm parameters has been highlighted for other polyatomic molecules [86].

Electron impact rate coefficients have also been calculated using Biagi cross sections [66] and compared with recent measurements by Morillo-Candas and co-authors [69]. In [69], dissociation rate coefficients are measured in a pulsed DC glow discharge at moderate values of $E / N$ (i.e. $45 \mathrm{Td}<$ $E / N<110 \mathrm{Td}$ ) and compared with other measurements by Corvin and Corrigan [87]. Moreover, in the same work, experimental results are compared with results from BOLSIG+ [22], where EEDFs are obtained from a solution of the twoterm Boltzmann equation using the IST-Lisbon cross sections set [28] and rate coefficients are calculated from several sets of cross sections from literature. Morillo-Candas and coauthors [69] recommend the use of Polak and Slovetsky cross sections [88] for calculations of electron impact dissociation rate coefficients in $\mathrm{CO}_{2}$, in the $E / N$ range investigated. In this work, EEDFs are calculated using cross sections of the Biagi database [66], while dissociation rate coefficients are calculated using both Polak and Slovetsky [88] and Biagi [66] cross sections. These last were not considered in [69]. In figure 14, total dissociation rate coefficients obtained with BOLSIG+ and $\mathrm{MCF}$ at $T_{\text {gas }}=300 \mathrm{~K}$ are compared with experimental results of Morillo-Candas and co-authors [69] and Corvin and Corrigan [87]. In the calculations, it is assumed that vibrational and rotational states are populated according to a Boltzmann distribution at $T_{\text {gas }}$. Since there are uncertainties on the measurements of Corvin and Corrigan [87] given by the total pressure change and the determination of gaseous dissociation products in the experiment, we focus on the comparison of our numerical results with measurements of Morillo-Candas and co-authors [69]. Moreover, for all $E / N$ values, large discrepancies between calculations and experimental measurements of Corvin and Corrigan [87] are found. The measurements of [87] are also significantly different than the ones of Morillo-Candas and co-authors and, in [69], possible causes of this discrepancy are pointed out. Overall, the agreement between measurements of Morillo-Candas and calculations using Biagi cross sections is relatively good up to around $60 \mathrm{Td}$, while, for higher $E / N$ values, results of the calculations are systematically higher than the same measurements.

MCF results suggest that the main dissociation channel, in this $E / N$ range, is through triplet excitation with thresholds of $8.89 \mathrm{eV}$ and $11.30 \mathrm{eV}$. A smaller contribution is given by dipole-allowed transitions with thresholds between 6.5-8.75 eV and 8.9-10.15 eV. For $E / N<70 \mathrm{Td}$, deviations between MCF and BOLSIG + results exceed $60 \%$, due to the anisotropy of the EVDF. Moreover, it is worth noting that BOLSIG + results are obtained with a modified input file of cross sections that takes into account superelastic collisions between vibrational states and the population fractions of those excited states and the same modified input file is used in MCF. Instead, if the file downloaded from LXCat is 


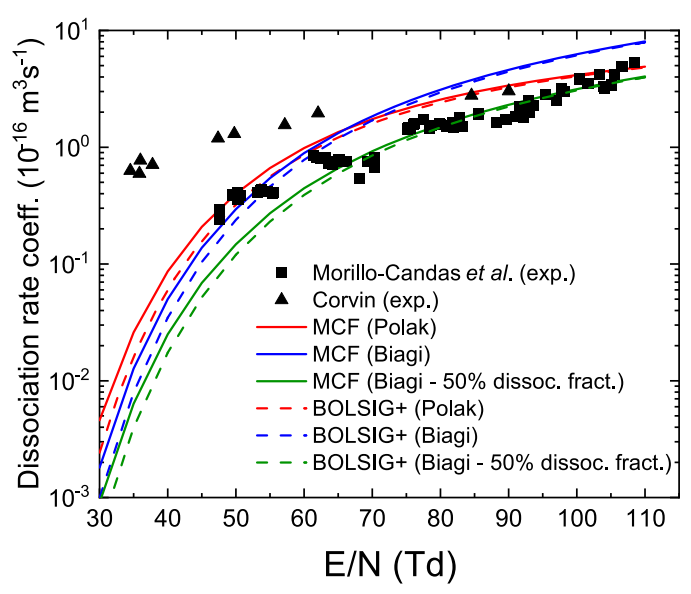

Figure 14. Electron impact dissociation rate coefficients measured $[69,87]$ and calculated with MCF and BOLSIG $+[22]$ as a function of reduced electric field.

used in BOLSIG + without purposely modifying it, larger discrepancies between MCF and BOLSIG+ are found due to the different treatment of the excited states population (not shown).

In figure 14, calculations using the cross sections of Polak and Slovetsky [88] are also shown. In those calculations, EEDFs are obtained from MCF and BOLSIG+ using only cross sections from the Biagi database, whereas the Polak and Slovetsky cross section is used for computation of rate coefficients. MCF and BOLSIG+ results obtained with Polak and Slovetsky cross sections at $E / N>70 \mathrm{Td}$ are relatively closer to experimental data of Morillo-Candas and co-authors, than the ones calculated with Biagi cross sections.

Different sources of uncertainties could affect the results. First of all, in calculations of dissociation rate coefficients with Biagi cross sections, excitation to singlet and triplets states between 6 and $12 \mathrm{eV}$ is assumed to be fully dissociative. An analysis of photoabsorption spectra suggests that those excitations are related to fast processes, like dissociation [68]. However, the dissociation fraction of those states is unknown. Moreover, excited states in molecules have a decay path which can also occur through vibrational relaxation and this can lead to a dissociation fraction below $100 \%$ [68]. In figure 14, MCF and BOLSIG+ calculations with Biagi cross sections, assuming a dissociation fraction of $50 \%$ from states having energies between 6 and $12 \mathrm{eV}$, is shown. This dissociation fraction is chosen to approximately match calculations at $300 \mathrm{~K}$ and experimental measurements at $E / N>60 \mathrm{Td}$. For lower $E / N$ values, discrepancies between MCF calculations and measurements of Morillo-Candas and co-authors [69] are found, while a better agreement is obtained with calculations at higher gas temperatures of about $700 \mathrm{~K}$ and by assuming the same value of dissociation fraction (not shown). Further investigation is needed in order to accurately estimate the $\mathrm{CO}_{2}$ dissociation fraction via dipole-allowed and triplet excitations. New calculations of electron impact cross sections, like the ones in [89], are very much sought, since measurements of those cross sections usually present large uncertainties.
In addition, it is important to mention that measurements in [69] have been performed in conditions of gas temperatures between 300 and $700 \mathrm{~K}$ [90]. In particular, results with $E / N<60 \mathrm{Td}$ are obtained at higher current and pressure and thus at higher gas temperatures [90]. This fact may explain the discrepancies between MCF calculations with Biagi cross sections at $300 \mathrm{~K}$ and $50 \%$ dissociation fraction and measurements in that range of $E / N$. Indeed, following the results of figure 11, rate coefficients are expected to increase with $T_{\text {gas }}$. Moreover, as regards gas composition, in experiments small fractions of dissociation products are formed [69] and those can have a large impact in EEDF calculations, as shown in [91]. For this reason, and in order to better estimate a correct value of dissociation fraction, experiments and calculations performed at different controlled gas temperatures are highly sought after. From the results of this analysis we conclude that, given the overall good agreement between measurements and calculations, without the introduction of an additional dissociation fraction, cross sections of Polak and Slovetsky [88] are preferred for calculations of the electron impact dissociation rate coefficients, in this $E / N$ range, as pointed out already in [69].

\section{Conclusions}

In this work, the MCF method has been benchmarked against two-term and multi-term solutions of the EBE for calculations of EVDFs in $\mathrm{CO}_{2}$, using the Biagi dataset of cross sections [66]. MCF results have been obtained by means of a fully native code developed by the authors [54]. The study of the $\mathrm{CO}_{2}$ molecule is particularly challenging, due to the presence of a broad Ramsauer minimum in the elastic momentum transfer cross sections and evenly distributed energy losses due to vibrational excitations. In this framework, MCF can potentially be considered as a reference method, faster than conventional MC approaches, for benchmarking popular Boltzmann solvers that are based on the two-term approximation, with the caveat that, as we have shown, each method must operate with its most appropriate optimized set of cross sections. This model has been integrated in a 0-D model for plasma chemical kinetics in [92].

The accuracy of MCF, with respect to the ten-term Boltzmann solver MultiBolt [35], has been assessed through calculations of EVDFs and of rate coefficients for vibrational excitation and dissociation mechanisms, finding an excellent agreement. As expected, large deviations from results obtained with the two-term solver BOLSIG $+[23]$ have been found at moderate $E / N$ (between 20 and $80 \mathrm{Td}$ ) for calculations of rate coefficients that are strongly sensitive to the tail of the distribution. This result shows the importance of the choice of data and methods to describe electron kinetics in plasma chemistry models that use these coefficients.

Subsequently, the MCF method has been upgraded to include the effect of the finite gas temperature of the background gas. This makes MCF more reliable for calculations at low $E / N$, where the cold gas approximation does not apply and thermal gas effects have an impact on the calculation of rate coefficients and swarm parameters. Results of MCF including finite gas temperature have been benchmarked against results 
of BOLSIG + [23], that includes a Davydov-Boltzmann correction in the elastic operator, showing excellent agreement at low fields.

Furthermore, we have extended the model to include the population of vibrational and rotational states of the ground electronic state. In this way, $\mathrm{CO}_{2}$ molecules are modelled as a real gas, instead of having their population collapsed into the $\mathrm{CO}_{2}(000)$ state. The inclusion of the population has an effect not only on superelastic collisions, but also on the elastic momentum transfer cross sections. It is important to notice that the detailed description of excited states and superelastic collisions is not explicitly presented in the cross sections set that is available in LXCat. However, their inclusion is fundamental for reproducing experimental transport coefficients at low $E / N$ with high accuracy. In addition, the Biagi cross sections set has been used for calculations of electron impact dissociation rate coefficients, showing good agreement with recent measurements [69], using the Polak and Slovetsky [88] cross sections for calculations of dissociation rate coefficients. The use of the Biagi cross sections set, that includes a detailed description of electron energy loss mechanisms, opens up possibilities for comparison with other cross sections sets. The need for dedicated experiments performed at different gas temperatures, to assess the influence of temperature and validate the results and hypotheses of models, has been highlighted.

The model will be extended to simulate electron distribution functions in time-dependent and space-dependent electric fields and to test anisotropic scattering in collision dynamics.

\section{Acknowledgments}

The authors are grateful to Dr. J van Dijk from Eindhoven University of Technology for useful discussions. This work is part of the Shell-NWO/FOM initiative 'Computational sciences for energy research' of Shell and Chemical Sciences, Earth and Life Sciences, Physical Sciences, FOM and STW.

\section{ORCID iDs}

L Vialetto (1D https://orcid.org/0000-0003-3802-8001

P Viegas (i) https://orcid.org/0000-0002-3820-3300

S Longo (i) https://orcid.org/0000-0002-5038-7659

P Diomede (D) https://orcid.org/0000-0002-4523-3049

\section{References}

[1] Capitelli M, Ferreira C M, Gordiets B F and Osipov A I 2013 Plasma Kinetics in Atmospheric Gases vol 31 (Berlin: Springer)

[2] Gordiets B F, Sobolev N N and Shelepin L A 1968 Kinetics of physical processes in $\mathrm{CO}_{2}$ lasers Sov. Phys. JETP 26 1039-45

[3] Judd O P 1974 The effect of gas mixture on the electron kinetics in the electrical $\mathrm{CO}_{2}$ gas laser J. Appl. Phys. 45 4572-5

[4] Chen Y and Li S 1993 Buckling failure of the axially precompressed cylindrical shell irradiated by $\mathrm{CW} \mathrm{CO}_{2}$ laser beam 24th Plasma Dynamics, and Lasers Conf. vol 3231
[5] Capezzuto P, Cramarossa F, D' Agostino R and Molinari E 1976 Contribution of vibrational excitation to the rate of carbon dioxide dissociation in electrical discharges J. Phys. Chem. 80 882-8

[6] Capitelli M and Pietanza L D 2019 Past and present aspects of Italian plasma chemistry Rend. Lincei Sci. Fis. Nat. 30 31-48

[7] Fridman A 2008 Plasma Chemistry (Cambridge: Cambridge University Press)

[8] Bongers $\mathrm{W}$ et al 2017 Plasma-driven dissociation of $\mathrm{CO}_{2}$ for fuel synthesis Plasma Process. Polym. 141600126

[9] Guerra V, Silva T, Ogloblina P, Grofulović M, Terraz L, Silva M L. d., Pintassilgo C D, Alves L L and Guaitella O 2017 The case for in situ resource utilisation for oxygen production on Mars by non-equilibrium plasmas Plasma Sources Sci. Technol. 26 11LT01

[10] Nighan W L 1970 Electron energy distributions and collision rates in electrically excited $\mathrm{N}_{2}, \mathrm{CO}$, and $\mathrm{CO}_{2}$ Phys. Rev. A 2 1989-2000

[11] White R D, Robson R E, Dujko S, Nicoletopoulos P and Li B 2009 Recent advances in the application of Boltzmann equation and fluid equation methods to charged particle transport in non-equilibrium plasmas J. Phys. D: Appl. Phys. 42 194001

[12] Makabe T and Petrović Z L 2014 Plasma Electronics: Applications in Microelectronic Device Fabrication (Boca Raton, FL: CRC Press)

[13] Robson R, White R and Hildebrandt M 2017 Fundamentals of Charged Particle Transport in Gases and Condensed Matter (Boca Raton, FL: CRC Press)

[14] Makabe T 2018 Velocity distribution of electrons in timevarying low-temperature plasmas: progress in theoretical procedures over the past 70 years Plasma Sources Sci. Technol. 27033001

[15] Capitelli M, Celiberto R, Colonna G, Esposito F, Gorse C, Hassouni K, Laricchiuta A and Longo S 2015 Fundamental Aspects of Plasma Chemical Physics: Kinetics vol 85 (Berlin: Springer)

[16] Kozák T and Bogaerts A 2014 Splitting of $\mathrm{CO}_{2}$ by vibrational excitation in non-equilibrium plasmas: a reaction kinetics model Plasma Sources Sci. Technol. 23045004

[17] Aerts R, Somers W and Bogaerts A 2015 Carbon dioxide splitting in a dielectric barrier discharge plasma: a combined experimental and computational study ChemSusChem $8702-16$

[18] Berthelot A and Bogaerts A 2017 Modeling of $\mathrm{CO}_{2}$ splitting in a microwave plasma: how to improve the conversion and energy efficiency J. Phys. Chem. C 121 8236-51

[19] Pancheshnyi S, Eismann B, Hagelaar G J M and Pitchford L C 2008 Computer Code ZDPlasKin (Toulouse, France: University of Toulouse, LAPLACE, CNRS-UPS-INP) http:// zdplaskin.laplace.univ-tlse.fr (last access 29/08/2019)

[20] Dorai R 2002 Modeling of atmospheric pressure plasma processing of gases and surfaces PhD Thesis University of Illinois at Urbana-Champaign

[21] Dorai R and Kushner M J 2000 Consequences of propene and propane on plasma remediation of NOx J. Appl. Phys. 88 3739-47

[22] http://bolsig.laplace.univ-tlse.fr/ (last access 29/08/2019)

[23] Hagelaar G J M and Pitchford L C 2005 Solving the Boltzmann equation to obtain electron transport coefficients and rate coefficients for fluid models Plasma Sources Sci. Technol. 14 722-33

[24] Pietanza L D, Colonna G, D’Ammando G, Laricchiuta A and Capitelli M 2016 Electron energy distribution functions and fractional power transfer in 'cold' and excited $\mathrm{CO}_{2}$ discharge and post discharge conditions Phys. Plasmas 23013515

[25] Pietanza L D, Colonna G, D'Ammando G and Capitelli M 2016 Time-dependent coupling of electron energy distribution function, vibrational kinetics of the asymmetric mode 
of $\mathrm{CO}_{2}$ and dissociation, ionization and electronic excitation kinetics under discharge and post-discharge conditions Plasma Phys. Control. Fusion 59014035

[26] Silva T, Grofulović M, Terraz L, Pintassilgo C D and Guerra V 2018 Modelling the input and relaxation of vibrational energy in CO2 plasmas J. Phys. D: Appl. Phys. 51464001

[27] Grofulović M, Silva T, Klarenaar B L M, Morillo-Candas A-S, Guaitella O, Engeln R, Pintassilgo C D and Guerra V 2018 Kinetic study of $\mathrm{CO}_{2}$ plasmas under non-equilibrium conditions. II. Input of vibrational energy Plasma Sources Sci. Technol. 27115009

[28] Grofulović M, Alves L L and Guerra V 2016 Electron-neutral scattering cross sections for $\mathrm{CO}_{2}$ : a complete and consistent set and an assessment of dissociation J. Phys. D: Appl. Phys. 49395207

[29] https://github.com/IST-Lisbon/LoKI (last access 29/08/2019)

[30] Tejero-del-Caz A, Guerra V, Gonçalves D, da Silva M L, Marques L, Pinhão N, Pintassilgo C D and Alves L L 2019 The LisbOn kinetics Boltzmann solver Plasma Sources Sci. Technol. 28043001

[31] White R D, Robson R E, Schmidt B and Morrison M A 2003 Is the classical two-term approximation of electron kinetic theory satisfactory for swarms and plasmas? J. Phys. D: Appl. Phys. 36 3125-31

[32] Winkler R, Braglia G L and Wilhelm J 1988 Impact of nonisotropic elastic and inelastic scattering on the electron velocity distribution in weakly ionized plasmas Il Nuovo Cimento D 10 1209-34

[33] Pitchford L C, ONeil S V and Rumble J R 1981 Extended Boltzmann analysis of electron swarm experiments Phys. Rev. A 23 294-304

[34] Ness K F and Robson R E 1986 Velocity distribution function and transport coefficients of electron swarms in gases. II. Moment equations and applications Phys. Rev. A 34 2185-209

[35] Stephens J 2018 A multi-term Boltzmann equation benchmark of electron-argon cross-sections for use in low temperature plasma models J. Phys. D: Appl. Phys. 51125203

[36] http://gitlab.com/LXCatThirdParty/MultiBolt (last access 03/09/2019)

[37] Loffhagen D 2016 Multi-term and non-local electron Boltzmann equation Plasma Modeling vol 2053-2563 (Bristol: IOP Publishing) pp 3-1-3-30

[38] White R D, Robson R and Ness K F 2001 Visualization of ion and electron velocity distribution functions in electric and magnetic fields J. Phys. D: Appl. Phys. 342205

[39] Segur P, Yousfi M and Bordage M C 1984 Comparisons between different methods of solution of the Boltzmann equation adapted to the calculation of swarm parameters in a weakly ionised medium J. Phys. D: Appl. Phys. 17 2199-214

[40] Vass M, Korolov I, Loffhagen D, Pinhão N and Donkó Z 2017 Electron transport parameters in $\mathrm{CO}_{2}$ : scanning drift tube measurements and kinetic computations Plasma Sources Sci. Technol. 26065007

[41] Longo S 2000 Monte Carlo models of electron and ion transport in non-equilibrium plasmas Plasma Sources Sci. Technol. 9 468-76

[42] Longo S 2006 Monte Carlo simulation of charged species kinetics in weakly ionized gases Plasma Sources Sci. Technol. 15 S181-8

[43] Dujko S, White R D, Petrović Z L and Robson R E 2011 A multiterm solution of the nonconservative Boltzmann equation for the analysis of temporal and spatial non-local effects in charged-particle swarms in electric and magnetic fields Plasma Sources Sci. Technol. 20024013

[44] Vojnović M M, Ristić M M, Stanković V V and Poparić G B 2019 Electron-induced vibrational excitation of $\mathrm{CO}_{2}$ in $\mathrm{dc}$ electric and magnetic fields Phys. Rev. E 99063211
[45] Tan X and Go D B 2019 Rational design of plasma-enhanced catalysis at microscale dimensions for the dissociation of CO2 J. Electrost. 97 71-4

[46] Biagi S F 1999 Monte Carlo simulation of electron drift and diffusion in counting gases under the influence of electric and magnetic fields Nucl. Instrum. Methods Phys. Res. A 421 234-40

[47] http://magboltz.web.cern.ch/magboltz/ (last access 02/08/2019)

[48] Braglia G L and Romanò L 1984 Monte Carlo and Boltzmann two-term calculations of electron transport in $\mathrm{CO}_{2}$ Lett. Nuovo Cimento 40 513-8

[49] Braglia G L, Wilhelm J and Winkler E 1985 Multi-term solutions of Boltzmann's equation for electrons in the real gases $\mathrm{Ar}, \mathrm{CH}_{4}$ and $\mathrm{CO}_{2}$ Lett. Nuovo Cimento 44 365-78

[50] Caflisch R E 1998 Monte Carlo and quasi-Monte Carlo methods Acta Numer. 7 1-49

[51] Dupree S A and Fraley S K 2002 A Monte Carlo Primer: A Practical Approach to Radiation Transport vol 1 (Berlin: Springer)

[52] Schaefer G and Hui P 1990 The Monte Carlo flux method $J$. Comput. Phys. 89 1-30

[53] Longo S and Capitelli M 1994 A simple approach to treat anisotropic elastic collisions in Monte Carlo calculations of the electron energy distribution function in cold plasmas Plasma Chem. Plasma Process. 14 1-13

[54] Vialetto L, Longo S and Diomede P 2019 Benchmark calculations for electron velocity distribution function obtained with Monte Carlo flux simulations Plasma Sources Sci. Technol. 28115015

[55] Wannier G H 1971 Derivation of the Davydov distribution from the Boltzmann equation Am. J. Phys. 39 281-5

[56] Lifshitz E M and Pitaevskii L P 1981 Physical Kinetics (Oxford: Elsevier Ltd. Butterworth-Heinemann)

[57] Longo S and Diomede P 2004 Monte Carlo modeling of gas phase ion transport under thermal gradients and external fields Eur. Phys. J. Appl. Phys. 26 177-85

[58] Van Kampen N G 1992 Stochastic Processes in Physics and Chemistry vol 1 (Amsterdam: Elsevier)

[59] www.lxcat.net (last access 29/08/2019)

[60] http://math.sci.hiroshima-u.ac.jp/\&tnqx2dc;m-mat/MT/emt .html (last access 29/08/2019)

[61] Matsumoto M and Nishimura T 1998 Mersenne twister ACM Trans. Model. Comput. Simul. 8 3-30

[62] Skullerud H R 1968 The stochastic computer simulation of ion motion in a gas subjected to a constant electric field J. Phys. D: Appl. Phys. 1 1567-8

[63] Boeuf J P and Marode E 1982 A Monte Carlo analysis of an electron swarm in a nonuniform field: the cathode region of a glow discharge in helium J. Phys. D: Appl. Phys. 15 2169-87

[64] Ristivojevic Z and Petrović Z L 2012 A Monte Carlo simulation of ion transport at finite temperatures Plasma Sources Sci. Technol. 21035001

[65] http://netlib.org/lapack/ (last access 29/08/2019)

[66] Biagi database www.lxcat.net retrieved on December 172019

[67] Berkowitz J 2012 Photoabsorption, Photoionization, and Photoelectron Spectroscopy (New York: Academic)

[68] Biagi S F 2020 private communication.

[69] Morillo-Candas A S, Silva T, Klarenaar B L M, Grofulović M, Guerra V and Guaitella O 2020 Electron impact dissociation of $\mathrm{CO}_{2}$ Plasma Sources Sci. Technol. 29 01LT01

[70] Yousfi M, Hennad A and Alkaa A 1994 Monte Carlo simulation of electron swarms at low reduced electric fields Phys. Rev. E 49 3264-73

[71] Yousfi M, de Urquijo J, Juárez A, Basurto E and HernándezAvila J L 2009 Electron swarm coefficients in $\mathrm{CO}_{2}-\mathrm{N}_{2}$ and $\mathrm{CO}_{2}-\mathrm{O}_{2}$ mixtures IEEE Trans. Plasma Sci. 37 764-72

[72] Opal C B, Peterson W K and Beaty E C 1971 Measurements of secondary-electron spectra produced by electron impact 
ionization of a number of simple gases J. Chem. Phys. $\mathbf{5 5}$ $4100-6$

[73] Šimko T 1997 Modélisation du transport ionique et électronique dans un gaz soumis à un champ électrique. Comparaison modèle-expérience pour l'hydrogène $P h D$ thesis Université de Paris-Sud

[74] Buckman S J, Elford M T and Newman D S 1987 Electron scattering from vibrationally excited $\mathrm{CO}_{2} J$. Phys. B: At. Mol. Phys. 20 5175-82

[75] Haddad G N and Elford M T 1979 Low-energy electron scattering cross sections in carbon dioxide J. Phys. B: At. Mol. Phys. 12 L743-6

[76] Gerjuoy E and Stein S 1955 Rotational excitation by slow electrons Phys. Rev. 97 1671-9

[77] Fox J L and Dalgarno A 1979 Electron energy deposition in carbon dioxide Planet. Space Sci. 27 491-502

[78] Dutton database www.lxcat.net retrieved on August 182020

[79] ETHZ database www.lxcat.net retrieved on August 182020

[80] Heidelberg database www.lxcat.net retrieved on August 182020

[81] LAPLACE database www.lxcat.net retrieved on August 18 2020

[82] UNAM database www.lxcat.net retrieved on August 182020

[83] Tanaka H, Ishikawa T, Masai T, Sagara T, Boesten L, Takekawa M, Itikawa Y and Kimura M 1998 Elastic collisions of lowto intermediate-energy electrons from carbon dioxide: experimental and theoretical differential cross sections Phys. Rev. A 57 1798-808

[84] Raju G G 2005 Gaseous Electronics: Theory and Practice (Boca Raton, FL: CRC Press)

[85] Phelps database www.lxcat.net retrieved on August 182020
[86] Pinhão N R, Loffhagen D, Vass M, Hartmann P, Korolov I, Dujko S, Bošnjaković D and Donkó Z 2020 Electron swarm parameters in $\mathrm{C}_{2} \mathrm{H}_{2}, \mathrm{C}_{2} \mathrm{H}_{4}$ and $\mathrm{C}_{2} \mathrm{H}_{6}$ : measurements and kinetic calculations Plasma Sources Sci. Technol. 29 045009

[87] Corvin K K and Corrigan S J B 1969 Dissociation of carbon dioxide in the positive column of a glow discharge J. Chem. Phys. 50 2570-4

[88] Polak L S and Slovetsky D I 1976 Electron impact induced electronic excitation and molecular dissociation Int. J. Radiat. Phys. Chem. 8 257-82

[89] Scarlett L H, Savage J S, Fursa D V, Bray I and Zammit M C 2020 Electron-scattering on molecular hydrogen: convergent close-coupling approach Eur. Phys. J. D 74 $1-9$

[90] Klarenaar B L M, Morillo-Candas A S, Grofulović M, van de Sanden M C M, Engeln R and Guaitella O 2019 Excitation and relaxation of the asymmetric stretch mode of $\mathrm{CO}_{2}$ in a pulsed glow discharge Plasma Sources Sci. Technol. 28 035011

[91] Ogloblina P, Tejero-del-Caz A, Guerra V and Alves L L 2020 Electron impact cross sections for carbon monoxide and their importance in the electron kinetics of $\mathrm{CO}_{2}-\mathrm{CO}$ mixtures Plasma Sources Sci. Technol. 29015002

[92] Viegas P, Vialetto L, Wolf A J, Peeters F, Groen P W C, Righart T W H, Bongers W, van de Sanden R and Diomede P 2020 Insight into contraction dynamics of microwave plasmas for $\mathrm{CO}_{2}$ conversion from plasma chemistry modelling Plasma Sources Sci. Technol. https://doi.org/10.1088/1361$6595 / \mathrm{abb} 41 \mathrm{c}$ 